\title{
DBpedia - A Large-scale, Multilingual Knowledge Base Extracted from Wikipedia
}

Editor(s): Name Surname, University, Country

Solicited review(s): Name Surname, University, Country

Open review(s): Name Surname, University, Country

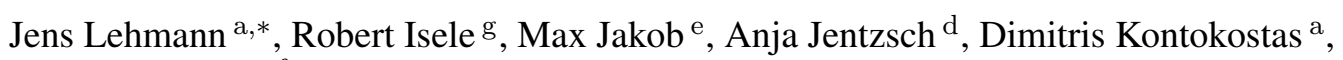

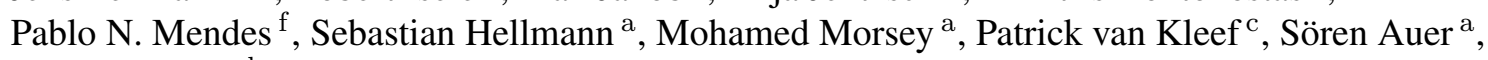 \\ Christian Bizer $b$

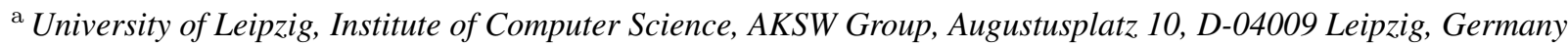 \\ E-mail: \{lastname\}@informatik.uni-leipzig.de \\ ${ }^{\mathrm{b}}$ University of Mannheim, Research Group Data and Web Science, B6-26, D-68159 Mannheim \\ E-mail: chris@informatik.uni-mannheim.de \\ ${ }^{\mathrm{c}}$ OpenLink Software, 10 Burlington Mall Road, Suite 265, Burlington, MA 01803, U.S.A. \\ E-mail: pkleef@openlinksw.com \\ ${ }^{\mathrm{d}}$ Hasso-Plattner-Institute for IT-Systems Engineering, Prof.-Dr.- Helmert-Str. 2-3, D-14482 Potsdam, Germany \\ E-mail: mail@anjajentzsch.de \\ ${ }^{\mathrm{e}}$ Neofonie GmbH, Robert-Koch-Platz 4, D-10115 Berlin, Germany \\ E-mail: max.jakob@neofonie.de \\ ${ }^{\mathrm{f}}$ Kno.e.sis - Ohio Center of Excellence in Knowledge-enabled Computing, Wright State University, Dayton, USA. \\ E-Mail: pablo@knoesis.org \\ ${ }^{g}$ Brox IT-Solutions GmbH, An der Breiten Wiese 9, D-30625 Hannover, Germany \\ E-Mail: mail@robertisele.com
}

\begin{abstract}
The DBpedia community project extracts structured, multilingual knowledge from Wikipedia and makes it freely available on the Web using Semantic Web and Linked Data technologies. The project extracts knowledge from 111 different language editions of Wikipedia. The largest DBpedia knowledge base which is extracted from the English edition of Wikipedia consists of over 400 million facts that describe 3.7 million things. The DBpedia knowledge bases that are extracted from the other 110 Wikipedia editions together consist of 1.46 billion facts and describe 10 million additional things. The DBpedia project maps Wikipedia infoboxes from 27 different language editions to a single shared ontology consisting of 320 classes and 1,650 properties. The mappings are created via a world-wide crowd-sourcing effort and enable knowledge from the different Wikipedia editions to be combined. The project publishes regular releases of all DBpedia knowledge bases for download and provides SPARQL query access to 14 out of the 111 language editions via a global network of local DBpedia chapters. In addition to the regular releases, the project maintains a live knowledge base which is updated whenever a page in Wikipedia changes. DBpedia sets 27 million RDF links pointing into over 30 external data sources and thus enables data from these sources to be used together with DBpedia data. Several hundred data sets on the Web publish RDF links pointing to DBpedia themselves and thus make DBpedia one of the central interlinking hubs in the Linked Open Data (LOD) cloud. In this system report, we give an overview of the DBpedia community project, including its architecture, technical implementation, maintenance, internationalisation, usage statistics and applications.
\end{abstract}

Keywords: Knowledge Extraction, Wikipedia, Multilingual Knowledge Bases, Linked Data, RDF 


\section{Introduction}

Wikipedia is the 6th most popular website 1 , the most widely used encyclopedia, and one of the finest examples of truly collaboratively created content. There are official Wikipedia editions in 287 different languages which range in size from a couple of hundred articles up to 3.8 million articles (English edition) ${ }^{2}$ Besides of free text, Wikipedia articles consist of different types of structured data such as infoboxes, tables, lists, and categorization data. Wikipedia currently offers only freetext search capabilities to its users. Using Wikipedia search, it is thus very difficult to find all rivers that flow into the Rhine and are longer than 100 miles, or all Italian composers that were born in the 18th century.

The DBpedia project builds a large-scale, multilingual knowledge base by extracting structured data from Wikipedia editions in 111 languages. This knowledge base can be used to answer expressive queries such as the ones outlined above. Being multilingual and covering an wide range of topics, the DBpedia knowledge base is also useful within further application domains such as data integration, named entity recognition, topic detection, and document ranking.

The DBpedia knowledge base is widely used as a testbed in the research community and numerous applications, algorithms and tools have been built around or applied to DBpedia. DBpedia is served as Linked Data on the Web. Since it covers a wide variety of topics and sets RDF links pointing into various external data sources, many Linked Data publishers have decided to set RDF links pointing to DBpedia from their data sets. Thus, DBpedia has developed into a central interlinking hub in the Web of Linked Data and has been a key factor for the success of the Linked Open Data initiative.

The structure of the DBpedia knowledge base is maintained by the DBpedia user community. Most importantly, the community creates mappings from Wikipedia information representation structures to the DBpedia ontology. This ontology - which will be explained in detail in Section 3- unifies different template structures, both within single Wikipedia language editions and across currently 27 different languages.

\footnotetext{
*Corresponding author. E-mail: lehmannn@informatik.unileipzig.de.

http://www.alexa.com/topsites Retrieved in October 2013 .

2 http://meta.wikimedia.org/wiki/List_of Wikipedias
}

The maintenance of different language editions of DBpedia is spread across a number of organisations. Each organisation is responsible for the support of a certain language. The local DBpedia chapters are coordinated by the DBpedia Internationalisation Committee.

The aim of this system report is to provide a description of the DBpedia community project, including the architecture of the DBpedia extraction framework, its technical implementation, maintenance, internationalisation, usage statistics as well as presenting some popular DBpedia applications. This system report is a comprehensive update and extension of previous project descriptions in [2] and [6]. The main advances compared to these articles are:

- The concept and implementation of the extraction based on a community-curated DBpedia ontology.

- The wide internationalisation of DBpedia.

- A live synchronisation module which processes updates in Wikipedia as well as the DBpedia ontology and allows third parties to keep their copies of DBpedia up-to-date.

- A description of the maintenance of public DBpedia services and statistics about their usage.

- An increased number of interlinked data sets which can be used to further enrich the content of DBpedia.

- The discussion and summary of novel third party applications of DBpedia.

Overall, DBpedia has undergone 7 years of continuous evolution. Table 17 provides an overview of the project's timeline.

The system report is structured as follows: In the next section, we describe the DBpedia extraction framework, which forms the technical core of DBpedia. This is followed by an explanation of the community-curated DBpedia ontology with a focus on multilingual support. In Section 4, we explicate how DBpedia is synchronised with Wikipedia with just very short delays and how updates are propagated to DBpedia mirrors employing the DBpedia Live system. Subsequently, we give an overview of the external data sets that are interlinked from DBpedia or that set RDF links pointing to DBpedia themselves (Section 5). In Section 6, we provide statistics on the usage of DBpedia and describe the maintenance of a large scale public data set. Within Section 7, we briefly describe several use cases and applications of DBpedia in a variety of different areas. Finally, we report on related work in Section 8 and give an outlook on the further development of DBpedia in Section 9 


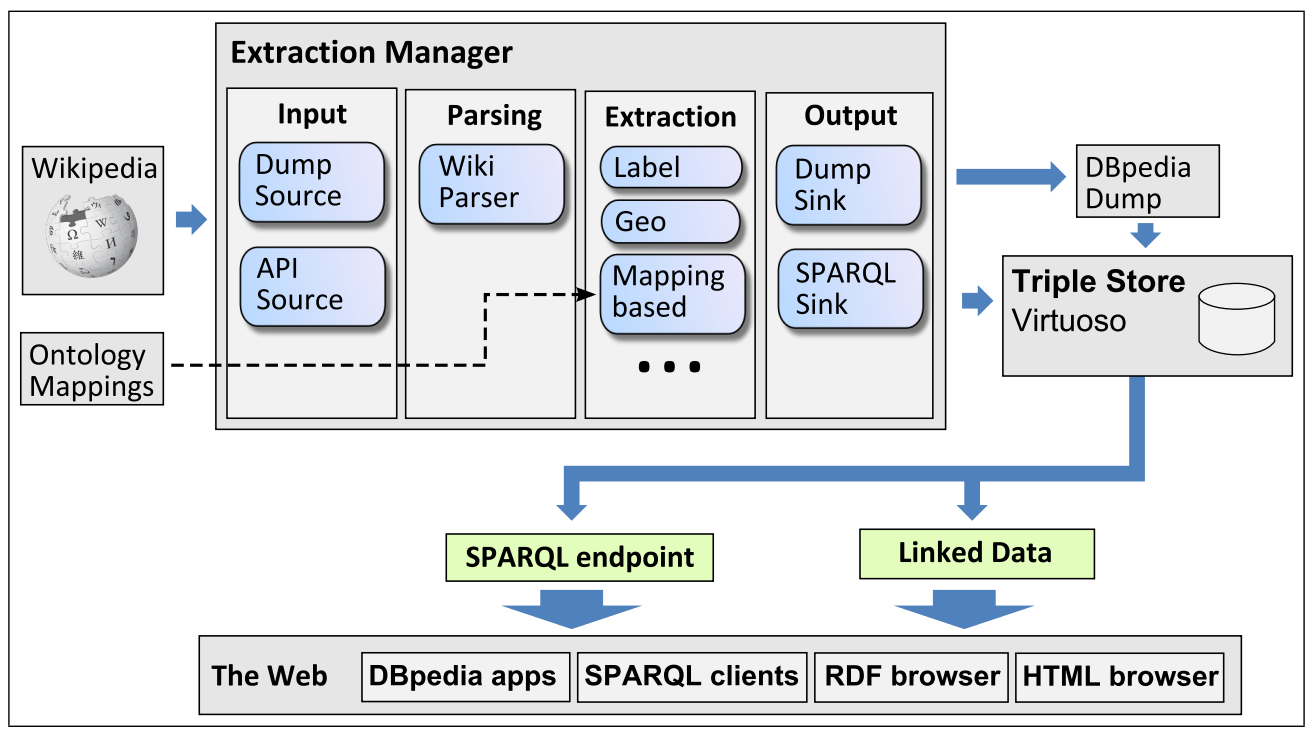

Fig. 1. Overview of DBpedia extraction framework.

\section{Extraction Framework}

Wikipedia articles consist mostly of free text, but also comprise of various types of structured information in the form of wiki markup. Such information includes infobox templates, categorisation information, images, geo-coordinates, links to external web pages, disambiguation pages, redirects between pages, and links across different language editions of Wikipedia. The DBpedia extraction framework extracts this structured information from Wikipedia and turns it into a rich knowledge base. In this section, we give an overview of the DBpedia knowledge extraction framework.

\subsection{General Architecture}

Figure 1 shows an overview of the technical framework. The DBpedia extraction is structured into four phases:

Input: Wikipedia pages are read from an external source. Pages can either be read from a Wikipedia dump or directly fetched from a MediaWiki installation using the MediaWiki API.

Parsing: Each Wikipedia page is parsed by the wiki parser. The wiki parser transforms the source code of a Wikipedia page into an Abstract Syntax Tree.

Extraction: The Abstract Syntax Tree of each Wikipedia page is forwarded to the extractors. DBpedia offers extractors for many different purposes, for instance, to extract labels, abstracts or geographical coordinates. Each extractor consumes an Abstract Syntax Tree and yields a set of RDF statements.
Output: The collected RDF statements are written to a sink. Different formats, such as N-Triples, are supported.

\subsection{Extractors}

The DBpedia extraction framework employs various extractors for translating different parts of Wikipedia pages to RDF statements. A list of all available extractors is shown in Table 1. DBpedia extractors can be divided into four categories:

Mapping-Based Infobox Extraction: The mappingbased infobox extraction uses manually written mappings that relate infoboxes in Wikipedia to terms in the DBpedia ontology. The mappings also specify a datatype for each infobox property and thus help the extraction framework to produce high quality data. The mapping-based extraction will be described in detail in Section 2.4

Raw Infobox Extraction: The raw infobox extraction provides a direct mapping from infoboxes in Wikipedia to RDF. As the raw infobox extraction does not rely on explicit extraction knowledge in the form of mappings, the quality of the extracted data is lower. The raw infobox data is useful if a specific infobox has not been mapped yet and thus is not available in the mapping-based extraction.

Feature Extraction: The feature extraction uses a number of extractors that are specialized in extracting a single feature from an article, such as a label or geographic coordinates. 
Statistical Extraction: Some NLP related extractors aggregate data from all Wikipedia pages in order to provide data that is based on statistical measures of page links or word counts, as further described in Section 2.6.

\subsection{Raw Infobox Extraction}

The type of Wikipedia content that is most valuable for the DBpedia extraction are infoboxes. Infoboxes are frequently used to list an article's most relevant facts as a table of attribute-value pairs on the top right-hand side of the Wikipedia page (for right-to-left languages on the top left-hand side). Infoboxes that appear in a Wikipedia article are based on a template that specifies a list of attributes that can form the infobox. A wide range of infobox templates are used in Wikipedia. Common examples are templates for infoboxes that describe persons, organisations or automobiles. As Wikipedia's infobox template system has evolved over time, different communities of Wikipedia editors use different templates to describe the same type of things (e.g. Infobox_city_japan, Infobox_swiss_town and Infobox_town_de). In addition, different templates use different names for the same attribute (e.g. birthplace and placeofbirth). As many Wikipedia editors do not strictly follow the recommendations given on the page that describes a template, attribute values are expressed using a wide range of different formats and units of measurement. An excerpt of an infobox that is based on a template for describing automobiles is shown below:

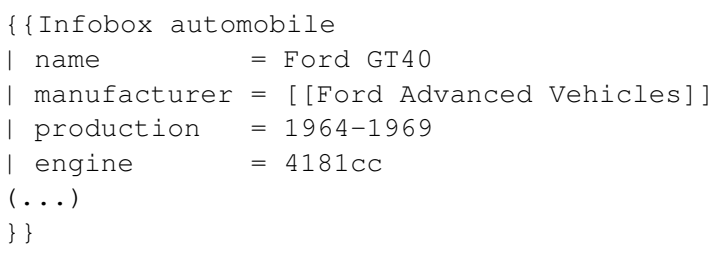

In this infobox, the first line specifies the infobox type and the subsequent lines specify various attributes of the described entity.

An excerpt of the extracted data is as follows:

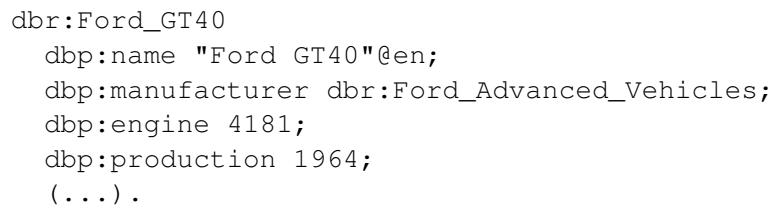

This extraction output has weaknesses: The resource is not associated to a class in the ontology and parsed values are cleaned up and assigned a datatyper based on heuristics. In particular, the raw infobox extractor searches for values in the following order: dates, coordinates, numbers, links and strings as default. Thus, the datatype assignment for the same property in different resources is non deterministic. The eng ine for example is extracted as a number but if another instance of the template used "cc4181" it would be extracted as string. This behaviour makes querying for properties in the $\mathrm{dbp}$ namespace inconsistent. Those problems can be overcome by the mapping-based infobox extraction presented in the next subsection.

\subsection{Mapping-Based Infobox Extraction}

In order to homogenize the description of information in the knowledge base, in 2010 a community effort was initiated to develop an ontology schema and mappings from Wikipedia infobox properties to this ontology. The alignment between Wikipedia infoboxes and the ontology is performed via community-provided mappings that help to normalize name variations in properties and classes. Heterogeneity in the Wikipedia infobox system, like using different infoboxes for the same type of entity or using different property names for the same property (cf. Section 2.3), can be alleviated in this way. This significantly increases the quality of the raw Wikipedia infobox data by typing resources, merging name variations and assigning specific datatypes to the values.

This effort is realized using the DBpedia Mappings Wik 3 , a MediaWiki installation set up to enable users to collaboratively create and edit mappings. These mappings are specified using the DBpedia Mapping Language. The mapping language makes use of MediaWiki templates that define DBpedia ontology classes and properties as well as template/table to ontology mappings. A mapping assigns a type from the DBpedia ontology to the entities that are described by the corresponding infobox. In addition, attributes in the infobox are mapped to properties in the DBpedia ontology. In the following, we show a mapping that maps infoboxes that use the Infobox_automobile template to the DBpedia ontology:

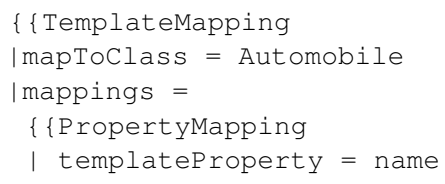

$\sqrt[3]{\text { http: //mappings.dbpedia.org }}$ 
Table 1

Overview of the DBpedia extractors (cf. Table 16 for a complete list of prefixes.).

\begin{tabular}{|c|c|c|}
\hline Name & Description & Example \\
\hline abstract & $\begin{array}{l}\text { Extracts the first lines of the } \\
\text { Wikipedia article. }\end{array}$ & $\begin{array}{l}\text { dbr:Berlin dbo:abstract "Berlin is the capital city of } \\
(\ldots) " \text {. }\end{array}$ \\
\hline article categories & $\begin{array}{l}\text { Extracts the categorization of the } \\
\text { article. }\end{array}$ & dbr:Oliver_Twist dc:subject dbr:Category:English_novels. \\
\hline category label & Extracts labels for categories. & dbr:Category:English_novels rdfs:label "English novels". \\
\hline category hierarchy & $\begin{array}{l}\text { Extracts information about which } \\
\text { concept is a category and how cat- } \\
\text { egories are related using the SKOS } \\
\text { Vocabulary. }\end{array}$ & $\begin{array}{l}\mathrm{dbr}: \text { Category }: \text { World_War_II skos:broader } \\
\mathrm{dbr}: \text { Category: Modern_history. }\end{array}$ \\
\hline disambiguation & Extracts disambiguation links. & dbr:Alien dbo:wikiPageDisambiguates dbr:Alien_(film). \\
\hline external links & $\begin{array}{l}\text { Extracts links to external web } \\
\text { pages related to the concept. }\end{array}$ & $\begin{array}{l}\text { dbr:Animal_Farm dbo:wikiPageExternalLink } \\
<\text { http://books.google.com/?id=RBGmrDnBs8UC> }\end{array}$ \\
\hline geo coordinates & Extracts geo-coordinates. & dbr:Berlin georss:point "52.5006 13.3989". \\
\hline grammatical gender & $\begin{array}{l}\text { Extracts grammatical genders for } \\
\text { persons. }\end{array}$ & dbr:Abraham_Lincoln foaf:gender "male". \\
\hline homepage & $\begin{array}{l}\text { Extracts links to the official home- } \\
\text { page of an instance. }\end{array}$ & dbr:Alabama foaf:homepage <http://alabama.gov/> \\
\hline image & $\begin{array}{l}\text { Extracts the first image of a } \\
\text { Wikipedia page. }\end{array}$ & dbr:Berlin foaf:depiction <http://../Overview_Berlin.jpg>. \\
\hline infobox & $\begin{array}{l}\text { Extracts all properties from all in- } \\
\text { foboxes. }\end{array}$ & dbr:Animal_Farm dbo:date "March 2010". \\
\hline interlanguage & Extracts interwiki links. & dbr:Albedo dbo:wikiPageInterLanguageLink dbr-de:Albedo. \\
\hline label & Extracts the article title as label. & dbr:Berlin rdfs:label "Berlin". \\
\hline lexicalizations & $\begin{array}{l}\text { Extracts information about surface } \\
\text { forms and their association with } \\
\text { concepts (only N-Quad format). }\end{array}$ & $\begin{array}{l}\text { dbr:Pine sptl:lexicalization lx:pine_tree ls:Pine_pine_tree } \\
\text { lx:pine_tree rdfs:label "pine tree". } \\
\text { ls:Pine_pine_tree sptl:puriGivensf "0.941" }\end{array}$ \\
\hline mappings & $\begin{array}{l}\text { Extraction based on mappings of } \\
\text { Wikipedia infoboxes to the DBpe- } \\
\text { dia ontology. }\end{array}$ & $\mathrm{dbr}$ :Berlin dbo: country dbr:Germany. \\
\hline page ID & Extracts page ids of articles. & dbr:Autism dbo:wikipageID "25" \\
\hline page links & $\begin{array}{l}\text { Extracts all links between } \\
\text { Wikipedia articles. }\end{array}$ & dbr:Autism dbo:wikiPageWikiLink dbr:Human_brain. \\
\hline persondata & $\begin{array}{l}\text { Extracts information about per- } \\
\text { sons represented using the Person- } \\
\text { Data template. }\end{array}$ & dbr:Andre_Agassi foaf:birthDate "1970-04-29". \\
\hline PND & $\begin{array}{l}\text { Extracts PND (Personennamen- } \\
\text { datei) data about a person. }\end{array}$ & dbr:William_Shakespeare dbo:individualisedPnd "118613723". \\
\hline redirects & $\begin{array}{l}\text { Extracts redirect links between ar- } \\
\text { ticles in Wikipedia. }\end{array}$ & $\begin{array}{l}\text { dbr:ArtificialLanguages dbo:wikiPageRedirects } \\
\mathrm{dbr}: \text { Constructed_language. }\end{array}$ \\
\hline revision ID & $\begin{array}{l}\text { Extracts the revision ID of the } \\
\text { Wikipedia article. }\end{array}$ & $\begin{array}{l}\text { dbr:Autism <http://www.w3.org/ns/prov\#wasDerivedFrom> } \\
\text { <http://en.wikipedia.org/wiki/Autism?oldid=495234324>. }\end{array}$ \\
\hline thematic concept & $\begin{array}{l}\text { Extracts 'thematic' concepts, the } \\
\text { centres of discussion for cate- } \\
\text { gories. }\end{array}$ & dbr:Category:Music skos:subject dbr:Music. \\
\hline $\begin{array}{l}\text { topic signatures } \\
\text { wiki page }\end{array}$ & $\begin{array}{l}\text { Extracts topic signatures. } \\
\text { Extracts links to corresponding ar- } \\
\text { ticles in Wikipedia. }\end{array}$ & $\begin{array}{l}\text { dbr:Alkane sptl:topicSignature "carbon alkanes atoms" . } \\
\text { dbr:AnAmericanInParis foaf:isPrimaryTopicof } \\
\text { <http://en.wikipedia.org/wiki/AnAmericanInParis>. }\end{array}$ \\
\hline
\end{tabular}




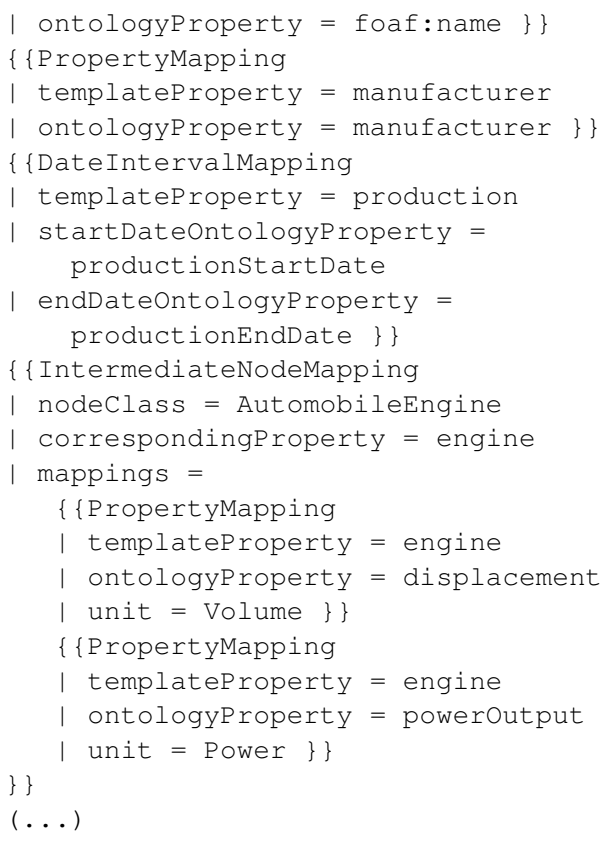

The RDF statements that are extracted from the previous infobox example are shown below. As we can see, the production period is correctly split into a start year and an end year and the engine is represented by a distinct RDF node. It is worth mentioning that all values are canonicalized to basic units. For example, in the engine mapping we state that engine is a Volume and thus, the extractor converts "4181cc" (cubic centimeters) to cubic meters ("0.004181"). Additionally, there can exist multiple mappings on the same property that search for different datatypes or different units. For example, a number with "PS" as a suffix for engine.

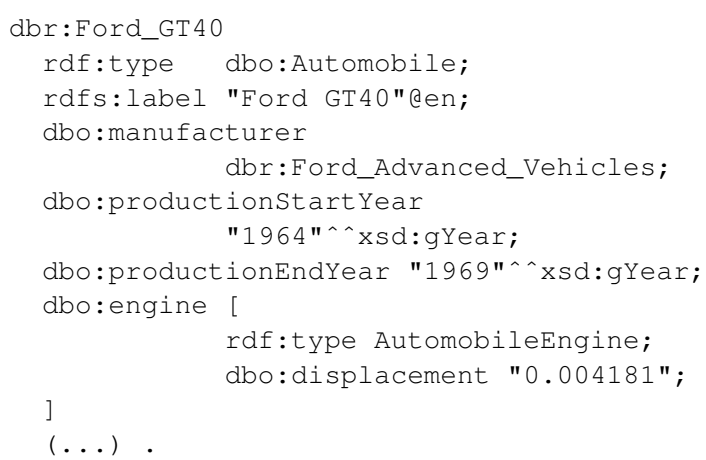

The DBpedia Mapping Wiki is not only used to map different templates within a single language edition of Wikipedia to the DBpedia ontology, but is used to map templates from all Wikipedia language editions to the shared DBpedia ontology. Figure 2 shows how the infobox properties author and $\sigma v \gamma \gamma \varrho \alpha \varphi \epsilon \alpha \varsigma-$ author in Greek - are both being mapped to the global identifier dbo: author. That means, in turn, that information from all language versions of DBpedia can be merged and DBpedias for smaller languages can be augmented with knowledge from larger DBpedias such as the English edition. Conversely, the larger DBpedia editions can benefit from more specialized knowledge from localized editions, such as data about smaller towns which is often only present in the corresponding language edition [43].

Besides hosting of the mappings and DBpedia ontology definition, the DBpedia Mappings Wiki offers various tools which support users in their work:

- Mapping Syntax Validator The mapping syntax validator checks for syntactic correctness and highlights inconsistencies such as missing property definitions.

- Extraction Tester The extraction tester linked on each mapping page tests a mapping against a set of example Wikipedia pages. This gives direct feedback about whether a mapping works and how the resulting data is structured.

- Mapping Tool The DBpedia Mapping Tool is a graphical user interface that supports users to create and edit mappings.

\subsection{URI Schemes}

For every Wikipedia article, the framework introduces a number of URIs to represent the concepts described on a particular page. Up to 2011 , DBpedia published URIs only under the http://dbpedia.org domain. The main namespaces were:

- http://dbpedia.org/resource/(prefix $d b r$ ) for representing article data. There is a oneto-one mapping between a Wikipedia page and a DBpedia resource based on the article title. For example, for the Wikipedia article on Berlin ${ }^{4}$ DBpedia will produce the URI $d b r$ :Berlin. Exceptions in this rule appear when intermediate nodes are extracted from the mapping-based infobox extractor as unique URIs (e.g., the engine mapping example in Section 2.4).

- http://dbpedia.org/property/(prefix $d b p$ ) for representing properties extracted from the raw infobox extraction (cf. Section 2.3), e.g. dbp:population.

4 http://en.wikipedia.org/wiki/Berlin 


\begin{tabular}{|c|c|c|c|c|}
\hline \multicolumn{3}{|c|}{ Mapping en:Infobox book } & \multicolumn{2}{|c|}{ Mapping el:Bıß入ío } \\
\hline \multicolumn{2}{|c|}{ Template Mapping (help) } & Class Book: & \multicolumn{2}{|c|}{ Template Mapping (help) } \\
\hline map to class & Book & & map to class & Book \\
\hline \multicolumn{2}{|l|}{ Mappings } & \multirow{2}{*}{$\begin{array}{l}\text { author } \\
\text { coverArtist } \\
\end{array}$} & \multicolumn{2}{|l|}{ Mappings } \\
\hline \multicolumn{2}{|c|}{ Property Mapping (help) } & & \multicolumn{2}{|c|}{ Property Mapping (help) } \\
\hline template property & author & firstPublicationDate & template property & 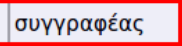 \\
\hline ontology property & author & illustrator & ontology property & author \\
\hline \multicolumn{2}{|c|}{ Property Mapping (help) } & isbn & \multicolumn{2}{|c|}{ Property Mapping (help) } \\
\hline template property & illustrator & lastPublicationDate & template property & हเкоvоүра́ \\
\hline ontology property & illustrator & $\ldots$ & ontology property & illustrator \\
\hline \multicolumn{2}{|c|}{$\{$ Infobox book } & & \multicolumn{2}{|l|}{$\{B i \beta \lambda i ́ o$} \\
\hline \multicolumn{2}{|c|}{ author = } & & 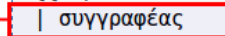 & $=$ \\
\hline \multicolumn{2}{|c|}{ title_orig = } & & हाoos & $=$ \\
\hline \multicolumn{2}{|c|}{ translator $=$} & & 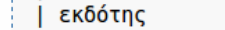 & $=$ \\
\hline \multicolumn{2}{|c|}{ illustrator } & & 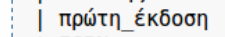 & $=$ \\
\hline \multicolumn{2}{|c|}{$\begin{array}{l}\text { subject } \\
\text { genre }\end{array}$} & & ISBN & $=$ \\
\hline \multicolumn{2}{|c|}{$\begin{array}{l}\text { | genre }= \\
\text { \}\} }\end{array}$} & & 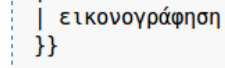 & $=$ \\
\hline
\end{tabular}

Fig. 2. Depiction of the mapping from the Greek and English Wikipedia templates about books to the same DBpedia Ontology class [24].

- http://dbpedia.org/ontology/(prefix $d b o$ ) for representing the DBpedia ontology (cf. Section 2.4, e.g. dbo:populationTotal.

Although data from other Wikipedia language editions were extracted, they used the same namespaces. This was achieved by exploiting the Wikipedia interlanguage links 5 . For every page in a language other than English, the page was extracted only if the page contained an inter-language link to an English page. In that case, using the English link, the data was extracted under the English resource name (i.e. dbr:Berlin).

Recent DBpedia internationalisation developments showed that this approach omitted valuable data [24]. Thus, starting from the DBpedia 3.7 releas 6 two types of data sets were generated. The localized data sets contain all things that are described in a specific language. Within the datasets, things are identified with language specific URIs such as http://<lang $>$. dbpedia. org/resource/ for article data and http:// <lang>.dbpedia.org/property/ for property data. In addition, we produce a canonicalized data set for each language. The canonicalized data sets only contain things for which a corresponding page in the English edition of Wikipedia exists. Within all canoni-

http://en.wikipedia.org/wiki/Help: Interlanguage_links

${ }^{\circ} \mathrm{A}$ list of all DBpedia releases is provided in Table 17 calized data sets, the same thing is identified with the same URI from the generic language-agnostic namespace http: / /dbpedia.org/resource/.

\subsection{NLP Extraction}

DBpedia provides a number of data sets which have been created to support Natural Language Processing (NLP) tasks [33]. Currently, four datasets are extracted: topic signatures, grammatical gender, lexicalizations and thematic concept. While the topic signatures and the grammatical gender extractors primarily extract data from the article text, the lexicalizations and thematic concept extractors make use of the wiki markup.

DBpedia entities can be referred to using many different names and abbreviations. The Lexicalization data set provides access to alternative names for entities and concepts, associated with several scores estimating the association strength between name and URI. These scores distinguish more common names for specific entities from rarely used ones and also show how ambiguous a name is with respect to all possible concepts that it can mean.

The topic signatures data set enables the description of DBpedia resources based on unstructured information, as compared to the structured factual data provided by the mapping-based and raw extractors. We build a Vector Space Model (VSM) where each DBpedia resource is a point in a multidimensional space of words. 
Each DBpedia resource is represented by a vector, and each word occurring in Wikipedia is a dimension of this vector. Word scores are computed using the tf-idf weight, with the intention to measure how strong is the association between a word and a DBpedia resource Note that word stems are used in this context in order to generalize over inflected words. We use the computed weights to select the strongest related word stems for each entity and build topic signatures [30].

There are two more Feature Extractors related to Natural Language Processing. The thematic concepts data set relies on Wikipedia's category system to capture the idea of a 'theme', a subject that is discussed in its articles. Many of the categories in Wikipedia are linked to an article that describes the main topic of that category. We rely on this information to mark DBpedia entities and concepts that are 'thematic', that is, they are the centre of discussion for a category.

The grammatical gender data set uses a simple heuristic to decide on a grammatical gender for instances of the class Person in DBpedia. While parsing an article in the English Wikipedia, if there is a mapping from an infobox in this article to the class dbo:Person, we record the frequency of gender-specific pronouns in their declined forms (Subject, Object, Possessive Adjective, Possessive Pronoun and Reflexive) - i.e. he, him, his, himself (masculine) and she, her, hers, herself (feminine). Grammatical genders for DBpedia entities are assigned based on the dominating gender in these pronouns.

\subsection{Summary of Other Recent Developments}

In this section we summarize the improvements of the DBpedia extraction framework since the publication of the previous DBpedia overview article [6] in 2009. One of the major changes on the implementation level is that the extraction framework has been rewritten in Scala in $2010^{7}$ to improve the efficiency of the extractors by an order of magnitude compared to the previous PHP based framework. The new more modular framework also allows to extract data from tables in Wikipedia pages and supports extraction from multiple MediaWiki templates per page. Another significant change was the creation and utilization of the DBpedia Mappings Wiki as described earlier. Further significant changes include the mentioned NLP extractors and the introduction of URI schemes.

Table 17 provides an overview of the project's evolution through time.
In addition, there were several smaller improvements and general maintenance: Overall, over the past four years, the parsing of the MediaWiki markup improved quite a lot which led to better overall coverage, for example, concerning references and parser functions. In addition, the collection of MediaWiki namespace identifiers for many languages is now performed semiautomatically leading to a high accuracy of detection. This concerns common title prefixes such as User, File, Template, Help, Portal etc. in English that indicate pages that do not contain encyclopedic content and would produce noise in the data. They are important for specific extractors as well, for instance, the category hierarchy data set is produced from pages of the Category namespace. Furthermore, the output of the extraction system now supports more formats and several compliance issues regarding URIs, IRIs, N-Triples and Turtle were fixed.

The individual data extractors have been improved as well in both number and quality in many areas. The abstract extraction was enhanced producing more accurate plain text representations of the beginning of Wikipedia article texts. More diverse and more specific datatypes do exist (e.g. many currencies and XSD datatypes such as xsd:gYearMonth, xsd:positiveInteger, etc.) and for a number of classes and properties, specific datatypes were added (e.g. inhabitants $/ \mathrm{km}^{2}$ for the population density of populated places and $\mathrm{m}^{3} / \mathrm{s}$ for the discharge of rivers). Many issues related to data parsers were resolved and the quality of the owl:sameAs data set for multiple language versions was increased by an implementation that takes bijective relations into account.

There are also further extractors, e.g. for Wikipedia page IDs and revisions. Moreover, redirect and disambiguation extractors were introduced and improved. For the redirect data, the transitive closure is computed while taking care of catching cycles in the links. The redirects also help regarding infobox coverage in the mapping-based extraction by resolving alternative template names. Moreover, in the PHP framework, if an infobox value pointed to a redirect, this redirection was not properly resolved and thus resulted in RDF links that led to URIs which did not contain any further information. Resolving redirects affected approximately $15 \%$ of all links, and hence increased the overall interconnectivity of resources in the DBpedia ontology.

Finally, a new heuristic to increase the connectiveness of DBpedia instances was introduced. If an infobox contains a string value that is not linked to another Wikipedia article, the extraction framework searches 


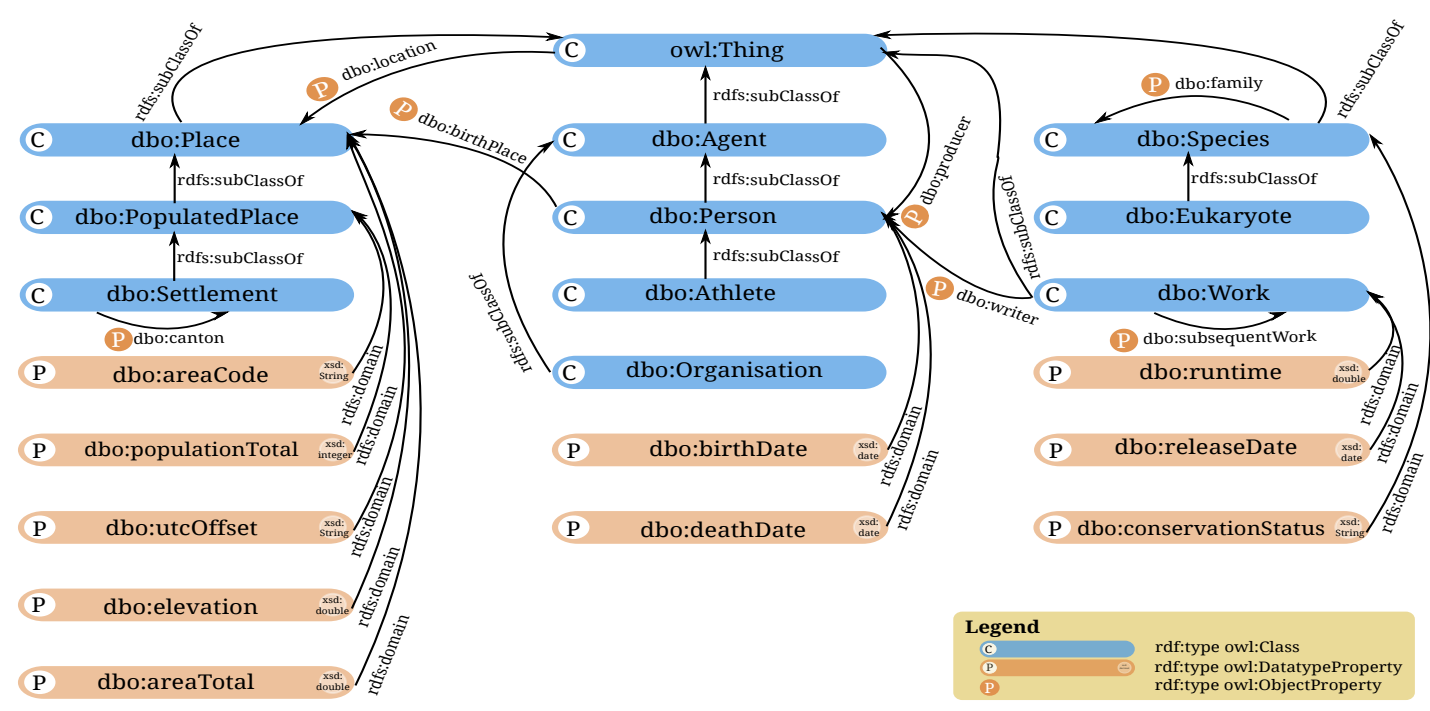

Fig. 3. Snapshot of a part of the DBpedia ontology.

for hyperlinks in the same Wikipedia article that have the same anchor text as the infobox value string. If such a link exists, the target of that link is used to replace the string value in the infobox. This method further increases the number of object property assertions in the DBpedia ontology.

Orthogonal to the previously mentioned improvements, there have been various efforts to assess the quality of the DBpedia datasets. [26] developed a framework for estimating the quality of DBpedia and a sample of 75 resources were analysed. A more comprehensive effort was performed in [48] by providing a distributed web-based interface [25] for quality assessment. In this study, 17 data quality problem types were analysed by 58 users covering 521 resources in DBpedia.

\section{DBpedia Ontology}

The DBpedia ontology consists of 320 classes which form a subsumption hierarchy and are described by 1,650 different properties. With a maximal depth of 5 , the subsumption hierarchy is intentionally kept rather shallow which fits use cases in which the ontology is visualized or navigated. Figure 3 depicts a part of the DBpedia ontology, indicating the relations among the top ten classes of the DBpedia ontology, i.e. the classes with the highest number of instances. The complete DBpedia ontology can be browsed online at http://mappings . dbpedia. org/server/ontology/classes/.

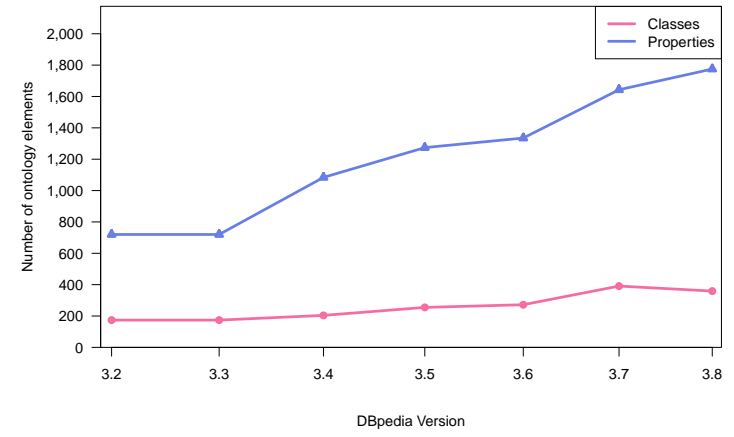

Fig. 4. Growth of the DBpedia ontology.

The DBpedia ontology is maintained and extended by the community in the DBpedia Mappings Wiki. Figure 4 depicts the growth of the DBpedia ontology over time. While the number of classes is not growing too much due to the already good coverage of the initial version of the ontology, the number of properties increases over time due to the collaboration on the DBpedia Mappings Wiki and the addition of more detailed information to infoboxes by Wikipedia editors.

\subsection{Mapping Statistics}

As of April 2013, there exist mapping communities for 27 languages, 23 of which are active. Figure 5 shows statistics for the coverage of these mappings in DBpedia. Figures (a) and (c) refer to the absolute number of template and property mappings that are defined for every DBpedia language edition. Figures (b) and (d) de- 

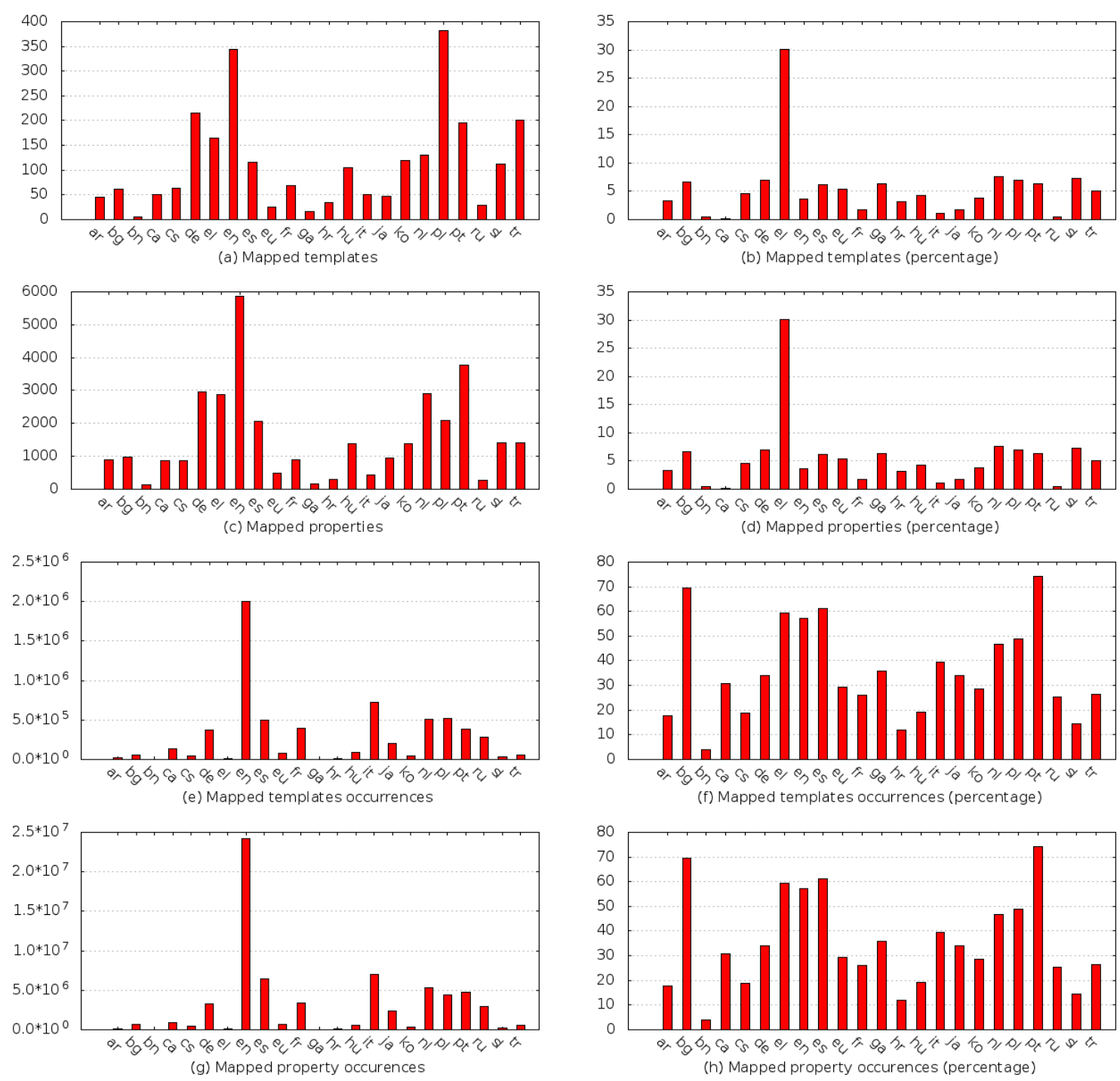

Fig. 5. Mapping coverage statistics for all mapping-enabled languages.

pict the percentage of the defined template and property mappings compared to the total number of available templates and properties for every Wikipedia language edition. Figures (e) and (g) show the occurrences (instances) that the defined template and property mappings have in Wikipedia. Finally, figures (f) and (h) give the percentage of the mapped templates and properties occurences, compared to the total templates and property occurences in a Wikipedia language edition.

It can be observed in the figure that the Portuguese DBpedia language edition is the most complete regarding mapping coverage. Other language editions such as Bulgarian, Dutch, English, Greek, Polish and Spanish have mapped templates covering more than $50 \%$ of total template occurrences. In addition, almost all languages have covered more than $20 \%$ of property occurrences, with Bulgarian and Portuguese reaching up to $70 \%$.

The mapping activity of the ontology enrichment process along with the editing of the ten most active mapping language communities is depicted in Figure 6 It is interesting to notice that the high mapping activity peaks coincide with the DBpedia release dates. For instance, the DBpedia 3.7 version was released on September 2011 and the 2nd and 3rd quarter of that year have a very high activity compared to the 4th quarter. In the last two years (2012 and 2013), most of the DBpedia mapping language communities have defined 


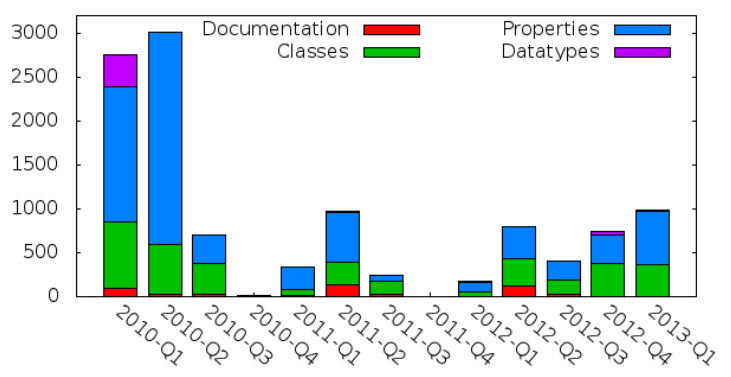

(a) Ontology edit activity

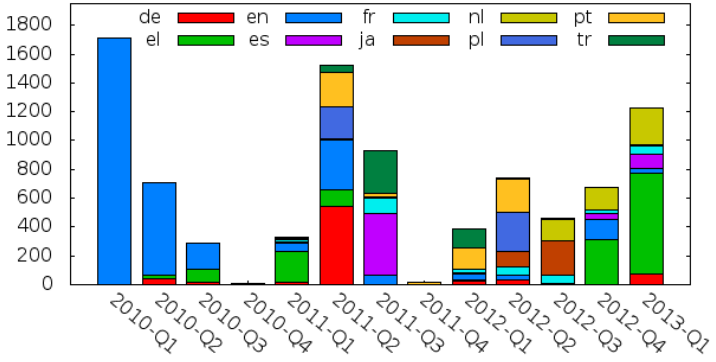

(b) Mapping edit activity for top 10 most active languages

Fig. 6. Mapping community activity for (a) ontology and (b) 10 most active language editions

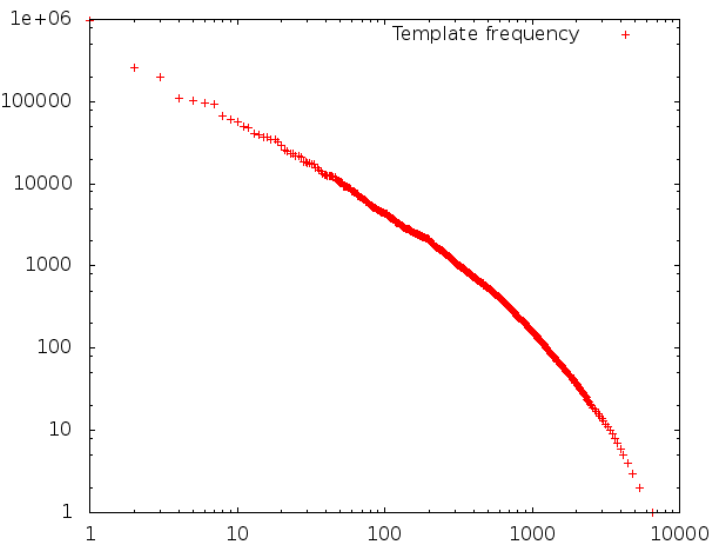

Fig. 7. English property mappings occurrence frequency (both axes are in log scale)

their own chapters and have their own release dates. Thus, recent mapping activity shows less fluctuation.

Finally, Figure 7 shows the English property mappings occurrence frequency. Both axes are in log scale and represent the number of property mappings ( $\mathrm{x}$ axis) that have exactly y occurrences (y axis). The occurrence frequency follows a long tail distribution. Thus, a low number of property mappings have a high number of occurrences and a high number of property mappings have a low number of occurences.

\subsection{Instance Data}

The DBpedia 3.8 release contains localized versions of DBpedia for 111 languages which have been extracted from the Wikipedia edition in the corresponding language. For 20 of these languages, we report in this section the overall number of entities being described by the localized versions as well as the number of facts (i.e. statements) that have been extracted from infoboxes describing these things. Afterwards, we report on the number of instances of popular classes within the 20 DBpedia versions as well as the conceptual overlap between the languages.

Table 2 shows the overall number of things, ontology and raw-infobox properties, infobox statements and type statements for the 20 languages. The column headings have the following meaning: $\mathrm{LD}=$ Localized data sets (see Section 2.5); CD = Canonicalized data sets (see Section 2.5; ; all = Overall number of instances in the data set, including instances without infobox data; with MD = Number of instances for which mappingbased infobox data exists; Raw Properties = Number of different properties that are generated by the raw infobox extractor; Mapping Properties = Number of different properties that are generated by the mappingbased infobox extractor; Raw Statements $=$ Number of statements (facts) that are generated by the raw infobox extractor; Mapping Statements $=$ Number of statements (facts) that are generated by the mapping-based infobox extractor.

It is interesting to see that the English version of DBpedia describes about three times more instances than the second and third largest language editions (French, German). Comparing the first column of the table with the second and third reveals which portion of the instances of a specific language correspond to instances in the English version of DBpedia and which portion of the instances is described by clean, mapping-based infobox data. The difference between the number of properties in the raw infobox data set and the cleaner mapping-based infobox data set (columns 4 and 5) results on the one hand from multiple Wikipedia infobox properties being mapped to a single ontology property. On the other hand, it reflects the number of mappings that have been so far created in the Mapping Wiki for a specific language.

Table 3 reports the number of instances for a set of popular classes from the third and forth hierarchy level 
Table 2

Basic statistics about Localized DBpedia Editions.

\begin{tabular}{|c|c|c|c|c|c|c|c|}
\hline & Inst. LD all & Inst. $C D$ all & Inst. with $M D C D$ & Raw Prop. $C D$ & Map. Prop. $C D$ & Raw Statem. $C D$ & Map. Statem. $C D$ \\
\hline en & $3,769,926$ & $3,769,926$ & $2,359,521$ & 48,293 & 1,313 & $65,143,840$ & $33,742,015$ \\
\hline de & $1,243,771$ & 650,037 & 204,335 & 9,593 & 261 & $7,603,562$ & $2,880,381$ \\
\hline it & 882,127 & 580,620 & 383,643 & 9,716 & 181 & $12,227,870$ & $4,804,731$ \\
\hline es & 879,091 & 542,524 & 310,348 & 14,643 & 476 & $7,740,458$ & $4,383,206$ \\
\hline $\mathrm{pt}$ & 699,446 & 460,258 & 272,660 & 12,851 & 602 & $6,255,151$ & $4,005,527$ \\
\hline $\mathrm{ca}$ & 367,362 & 241,534 & 112,934 & 8,696 & 183 & $3,689,870$ & $1,301,868$ \\
\hline cs & 225,133 & 148,819 & 34,893 & 5,564 & 334 & $1,857,230$ & 474,459 \\
\hline hu & 209,180 & 138,998 & 63,441 & 6,821 & 295 & $2,506,399$ & 601,037 \\
\hline $\mathrm{eu}$ & 132,877 & 108,713 & 41,401 & 2,245 & 19 & $2,255,897$ & 532,709 \\
\hline $\mathrm{sl}$ & 129,834 & 73,099 & 22,036 & 4,235 & 470 & $1,213,801$ & 222,447 \\
\hline bg & 125,762 & 87,679 & 38,825 & 3,984 & 274 & 774,443 & 488,678 \\
\hline $\mathrm{hr}$ & 109,890 & 71,469 & 10,343 & 3,334 & 158 & 701,182 & 151,196 \\
\hline el & 71,936 & 48,260 & 10,813 & 2,866 & 288 & 206,460 & 113,838 \\
\hline
\end{tabular}

of the ontology within the canonicalized DBpedia data sets for each language. The indented classes are subclasses of the superclasses set in bold. The zero values in the table indicate that no infoboxes have been mapped to a specific ontology class within the corresponding language so far. Again, the English version of DBpedia covers by far the most instances.

Table 4 shows, for the canonicalized, mapping-based data set, how many instances are described in multiple languages. The Instances column contains the total number of instances per class across all 20 languages, the second column contains the number of instances that are described only in a single language version, the next column contains the number of instances that are contained in two languages but not in three or more languages, etc. For example, 12,936 persons are described in five languages but not in six or more languages. The number 871,630 for the class Person means that all 20 language versions together describe 871,630 different persons. The number is higher than the number of persons described in the canonicalized English infobox data set $(763,643)$ listed in Table 3 , since there are infoboxes in non-English articles describing a person without a corresponding infobox in the English article describing the same person. Summing up columns 2 to $10+$ for the Person class, we see that 195,263 persons are described in two or more languages. The large difference of this number compared to the total number of
871,630 persons is due to the much smaller size of the localized DBpedia versions compared to the English one (cf. Table 2).

\subsection{Internationalisation Community}

The introduction of the mapping-based infobox extractor alongside live synchronisation approaches in [20] allowed the international DBpedia community to easily define infobox-to-ontology mappings. As a result of this development, there are presently mappings for 27 language $\mathrm{s}^{8}$ The DBpedia 3.7 releas $\mathrm{g}^{8}$ in September 2011 was the first DBpedia release to use the localized I18n (Internationalisation) DBpedia extraction framework [24].

At the time of writing, DBpedia chapters for 14 languages have been founded: Basque, Czech, Dutch, English, French, German, Greek, Italian, Japanese, Korean, Polish, Portuguese, Russian and Spanish ${ }^{10} \mathrm{Be}-$

\footnotetext{
${ }^{8}$ Arabic (ar), Bulgarian (bg), Bengali (bn), Catalan (ca), Czech (cs), German (de), Greek (el), English (en), Spanish (es), Estonian (et), Basque (eu), French (fr), Irish (ga), Hindi (hi), Croatian (hr), Hungarian (hu), Indonesian (id), Italian (it), Japanese (ja), Korean (ko), Dutch (nl), Polish (pl), Portuguese (pt), Russian (ru), Slovene (sl), Turkish (tr), Urdu (ur)

http://blog.dbpedia.org/2011/09/11/

${ }^{10}$ Accessed on 25/09/2013: http://wiki.dbpedia.org/ Internationalization/Chapters
} 
Table 3

Number of instances per class within 10 localized DBpedia versions.

\begin{tabular}{|c|c|c|c|c|c|c|c|c|c|c|}
\hline & en & it & $p l$ & es & $p t$ & $f r$ & $d e$ & $r u$ & $c a$ & $h u$ \\
\hline Person & 763,643 & 145,060 & 70,708 & 65,337 & 43,057 & 62,942 & 33,122 & 18,620 & 7,107 & 15,529 \\
\hline Athlete & 185,126 & 47,187 & 30,332 & 19,482 & 14,130 & 21,646 & 31,237 & 0 & 721 & 4,527 \\
\hline Artist & 61,073 & 12,511 & 16,120 & 25,992 & 10,571 & 13,465 & 0 & 0 & 2,004 & 3,821 \\
\hline Politician & 23,096 & 0 & 8,943 & 5,513 & 3,342 & 0 & 0 & 12,004 & 1,376 & 760 \\
\hline Place & 572,728 & 14,1101 & 182,727 & 132,961 & 116,660 & 80,602 & 131,766 & 67,932 & 73,078 & 18,324 \\
\hline Popul.Place & 387,166 & 138,077 & 167,034 & 121,204 & 109,418 & 72,252 & 79,410 & 63,826 & 72,743 & 15,535 \\
\hline Building & 60,514 & 1,270 & 2,946 & 3,570 & 803 & 921 & 83 & 43 & 0 & 527 \\
\hline River & 24,267 & 0 & 1,383 & 2 & 4,149 & 3,333 & 6,707 & 3,924 & 0 & 565 \\
\hline Organisation & 192,832 & 4,142 & 12,193 & 11,710 & 10,949 & 17,513 & 16,973 & 1,598 & 1,399 & 3,993 \\
\hline Company & 44,516 & 4,142 & 2,566 & 975 & 1,903 & 5,832 & 7,200 & 0 & 440 & 618 \\
\hline Educ.Inst. & 42,270 & 0 & 599 & 1,207 & 270 & 1,636 & 2,171 & 1,010 & 0 & 115 \\
\hline Band & 27,061 & 0 & 2,993 & 0 & 4,476 & 3,868 & 5,368 & 0 & 263 & 802 \\
\hline Work & 333,269 & 51,918 & 32,386 & 36,484 & 33,869 & 39,195 & 18,195 & 34,363 & 5,240 & 9,777 \\
\hline Music.Work & 159,070 & 23,652 & 14,987 & 15,911 & 17,053 & 16,206 & 0 & 6,588 & 697 & 4,704 \\
\hline Film & 71,715 & 17,210 & 9,467 & 9,396 & 8,896 & 9,741 & 15,038 & 12,045 & 3,859 & 2,320 \\
\hline Software & 27,947 & 5,682 & 3,050 & 4,833 & 3,419 & 5,733 & 2,368 & 0 & 606 & 857 \\
\hline
\end{tabular}

Table 4

Cross-language overlap: Number of instances that are described in multiple languages.

\begin{tabular}{l|r|r|r|r|r|r|r|r|r|r|r} 
Class & Instances & 1 & 2 & 3 & 4 & 5 & 6 & 7 & 8 & 9 & $10+$ \\
\hline Person & 871.630 & 676.367 & 94.339 & 42.382 & 21.647 & 12.936 & 8.198 & 5.295 & 3.437 & 2.391 & 4.638 \\
Place & 643.260 & 307.729 & 150.349 & 45.836 & 36.339 & 20.831 & 13.523 & 20.808 & 31.422 & 11.262 & 5.161 \\
Organisation & 206.670 & 160.398 & 22.661 & 9.312 & 5.002 & 3.221 & 2.072 & 1.421 & 928 & 594 & 1.061 \\
Work & 360.808 & 243.706 & 54.855 & 23.097 & 12.605 & 8.277 & 5.732 & 4.007 & 2.911 & 1.995 & 3.623
\end{tabular}

sides providing mappings from infoboxes in the corresponding Wikipedia editions, DBpedia chapters organise a local community and provide hosting for data sets and associated services.

While at the moment chapters are defined by ownership of the IP and server of the sub domain A record (e.g. http://ko.dbpedia.org) given by DBpedia maintainers, the DBpedia internationalisation committee ${ }^{11}$ is manifesting its structure and each language edition has a representative with a vote in elections. In some cases (e.g. Greek $\sqrt{12}$ and Dutch ${ }^{13}$ ) the existence of a local DBpedia chapter has had a positive effect on the creation of localized LOD clouds [24].

In the weeks leading to a new release, the DBpedia project organises a mapping sprint, where communities from each language work together to improve mappings, increase coverage and detect bugs in the extraction process. The progress of the mapping effort

\footnotetext{
11 http://wiki.dbpedia.org/ Internationalization

í/http://el.dbpedia.org

13 http://nl.dbpedia.org
}

is tracked through statistics on the number of mapped templates and properties, as well as the number of times these templates and properties occur in Wikipedia. These statistics provide an estimate of the coverage of each Wikipedia edition in terms of how many entities will be typed and how many properties from those entities will be extracted. Therefore, they can be used by each language edition to prioritize properties and templates with higher impact on the coverage.

The mapping statistics have also been used as a way to promote a healthy competition between language editions. A sprint page was created with bar charts that show how close each language is from achieving total coverage (as shown in Figure 5, and line charts showing the progress over time highlighting when one language is overtaking another in their race for higher coverage. The mapping sprints have served as a great motivator for the crowd-sourcing efforts, as it can be noted from the increase in the number of mapping contributions in the weeks leading to a release. 


\section{Live Synchronisation}

Wikipedia articles are continuously revised at a very high rate, e.g. the English Wikipedia, in June 2013, has approximately 3.3 million edits per month which is equal to 77 edits per minute ${ }^{14}$. This high change frequency leads to DBpedia data quickly being outdated, which in turn leads to the need for a methodology to keep DBpedia in synchronisation with Wikipedia. As a consequence, the DBpedia Live system was developed, which works on a continuous stream of updates from Wikipedia and processes that stream on the fly [20 36]. It allows extracted data to stay up-to-date with a small delay of at most a few minutes. Since the English Wikipedia is the largest among all Wikipedia editions with respect to the number of articles and the number of edits per month, it was the first language DBpedia Live supported ${ }^{15}$ Meanwhile, DBpedia Live for Dutch ${ }^{16}$ was developed.

\subsection{DBpedia Live System Architecture}

In order for live synchronisation to be possible, we need access to the changes made in Wikipedia. The Wikimedia foundation kindly provided us access to their update stream using the OAI-PMH protocol [27]. This protocol allows a programme to pull page updates in XML via HTTP. A Java component, serving as a proxy, constantly retrieves new updates and feeds them to the DBpedia Live framework. This proxy is necessary to decouple the stream from the framework to simplify maintenance of the software. The live extraction workflow uses this update stream to extract new knowledge upon relevant changes in Wikipedia articles.

The overall architecture of DBpedia Live is indicated in Figure 8. The major components of the system are as follows:

- Local Wikipedia Mirror: A local copy of a Wikipedia language edition is installed which is kept in real-time synchronisation with its live version using the OAI-PMH protocol. Keeping a local Wikipedia mirror allows us to exceed any access limits posed by Wikipedia.

- Mappings Wiki: The DBpedia Mappings Wiki, described in Section 2.4, serves as secondary input

\footnotetext{
14 http://stats.wikimedia.org/EN/SummaryEN. htm

if http://live.dbpedia.org

16 http://live.nl.dbpedia.org
}

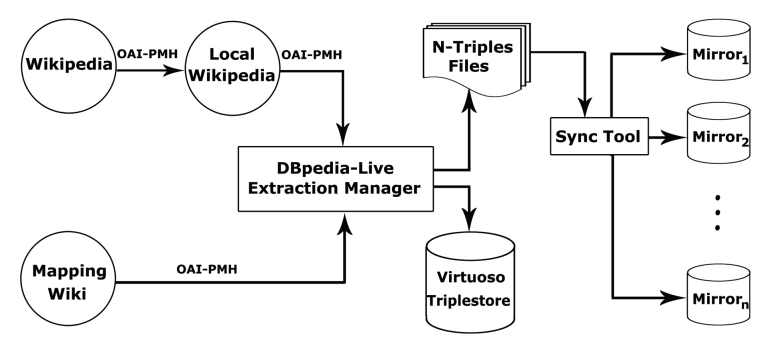

Fig. 8. Overview of DBpedia Live extraction framework.

source. Changes of the mappings wiki are also consumed via an OAI-PMH stream. Note that a single mapping change can affect a high number of DBpedia resources.

- DBpedia Live Extraction Manager: This is the core component of the DBpedia Live extraction architecture. The manager takes feeds of pages for re-processing as input and applies all the enabled extractors. After processing a page, the extracted triples are a) inserted into a backend triple store (in our case Virtuoso [10]), updating the old triples and b) saved as changesets into a compressed $\mathrm{N}$ Triples file structure.

- Synchronisation Tool: This tool allows third parties to keep DBpedia Live mirrors up-to-date by harvesting the produced changesets.

\subsection{Features of DBpedia Live}

The core components of the DBpedia Live Extraction framework provide the following features:

- Mapping-Affected Pages: The update of all pages that are affected by a mapping change.

- Unmodified Pages: The update of unmodified pages at regular intervals.

- Changesets Publication: The publication of triplechangesets.

- Synchronisation Tool: A synchronisation tool for harvesting updates to DBpedia Live mirrors.

- Data Isolation: Separate data from different sources.

Mapping-Affected Pages: Whenever an infobox mapping change occurs, all the Wikipedia pages that use that infobox are reprocessed. Taking Figure 2 as an example, if a new property mapping is introduced (i.e. dbo:translator) or an existing (i.e. dbo:illustrator) is updated or deleted, then all entities belonging to the class $d b o$ :Book are reprocessed. Thus, upon a mapping change, we identify all the affected Wikipedia pages and feed them for reprocessing. 
Unmodified Pages: Extraction framework improvements or activation / deactivation of DBpedia extractors might never be applied to rarely modified pages. To overcome this problem, we obtain a list of the pages which have not been processed over a period of time (30 days in our case) and feed that list to the DBpedia Live extraction framework for reprocessing. This feed has a lower priority than the update or the mapping affected pages feed and ensures that all articles reflect a recent state of the output of the extraction framework.

Publication of Changesets: Whenever a Wikipedia article is processed, we get two disjoint sets of triples. A set for the added triples, and another set for the deleted triples. We write those two sets into N-Triples files, compress them, and publish the compressed files as changesets. If another DBpedia Live mirror wants to synchronise with the DBpedia Live endpoint, it can just download those files, decompress and integrate them.

Synchronisation Tool: The synchronisation tool enables a DBpedia Live mirror to stay in synchronisation with our live endpoint. It downloads the changeset files sequentially, decompresses them and updates the target SPARQL endpoint via insert and delete operations.

Data Isolation: In order to keep the data isolated, DBpedia Live keeps different sources of data in different SPARQL graphs. Data from the article update feeds are contained in the graph with the URI http: / / I ive.dbpedia.org, static data (i.e. links to the LOD cloud) are kept in http://static. dopedia.org and the DBpedia ontology is stored in http://dbpedia.org/ontology. All data is also accessible under the http://dbpedia.org graph for combined queries. Next versions of DBpedia Live will also separate data from the raw infobox extraction and mapping-based infobox extraction.

\section{Interlinking}

DBpedia is interlinked with numerous external data sets following the Linked Data principles. In this section, we give an overview of the number and types of outgoing links that point from DBpedia into other data sets, as well as the external data sets that set links pointing to DBpedia resources.

\subsection{Outgoing Links}

Similar to the DBpedia ontology, DBpedia also follows a community approach for adding links to other third party data sets. The DBpedia project maintains a link repository ${ }^{17}$ for which conventions for adding linksets and linkset metadata are defined. The adherence to those guidelines is supervised by a linking committee. Linksets which are added to the repository are used for the subsequent official DBpedia release as well as for DBpedia Live. Table 5 lists the linksets created by the DBpedia community as of April 2013. The first column names the data set that is the target of the links. The second and third column contain the predicate that is used for linking as well as the overall number of links that is set between DBpedia and the external data set. The last column names the tool that was used to generate the links. The value S refers to Silk, L to LIMES, $\mathrm{C}$ to custom script and a missing entry means that the dataset is copied from the previous releases and not regenerated.

An example for the usage of links is the combination of data about European Union project funding (FTS) [32] and data about countries in DBpedia. The query below compares funding per year (from FTS) and country with the gross domestic product of a country (from DBpedia) ${ }^{18}$.

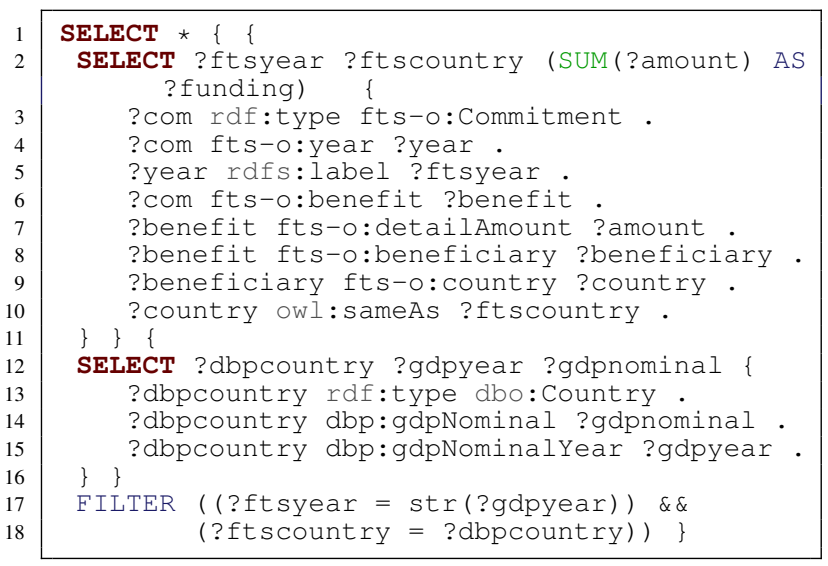

In addition to providing outgoing links on an instance-level, DBpedia also sets links on schemalevel pointing from the DBpedia ontology to equivalent terms in other schemas. Links to other schemata can be set by the community within the DBpedia Mappings Wiki by using owl :equivalentClass in class templates and owl : equivalentProperty in datatype or object property templates, respectively. In particular, in 2011 Google, Microsoft, and Yahoo! announced their collaboration on Schema.org, a col-

\footnotetext{
17 https://github.com/dbpedia/dbpedia-links

${ }^{18}$ Endpoint: http://fts.publicdata.eu/sparql Results: http://bit.1y/1c2mIwQ
} 
Table 5

Data sets linked from DBpedia

\begin{tabular}{|c|c|c|c|}
\hline Data set & Predicate & Count & Tool \\
\hline Amsterdam Museum & owl:sameAs & 627 & $\mathrm{~S}$ \\
\hline BBC Wildlife Finder & owl:sameAs & 444 & $\mathrm{~S}$ \\
\hline Book Mashup & $\begin{array}{l}\text { rdf:type } \\
\text { owl:sameAs }\end{array}$ & 9100 & \\
\hline Bricklink & dc:publisher & 10100 & \\
\hline CORDIS & owl:sameAs & 314 & $\mathrm{~S}$ \\
\hline Dailymed & owl:sameAs & 894 & $\mathrm{~S}$ \\
\hline DBLP Bibliography & owl:sameAs & 196 & $\mathrm{~S}$ \\
\hline DBTune & owl:sameAs & 838 & S \\
\hline Diseasome & owl:sameAs & 2300 & $\mathrm{~S}$ \\
\hline Drugbank & owl:sameAs & 4800 & $\mathrm{~S}$ \\
\hline EUNIS & owl:sameAs & 3100 & $\mathrm{~S}$ \\
\hline Eurostat (Linked Stats) & owl:sameAs & 253 & $\mathrm{~S}$ \\
\hline Eurostat (WBSG) & owl:sameAs & 137 & \\
\hline CIA World Factbook & owl:sameAs & 545 & $\mathrm{~S}$ \\
\hline flickr wrappr & dbp:hasPhoto- & 3800000 & $\mathrm{C}$ \\
\hline & Collection & & \\
\hline Freebase & owl:sameAs & 3600000 & $\mathrm{C}$ \\
\hline GADM & owl:sameAs & 1900 & \\
\hline GeoNames & owl:sameAs & 86500 & $\mathrm{~S}$ \\
\hline GeoSpecies & owl:sameAs & 16000 & $\mathrm{~S}$ \\
\hline $\mathrm{GHO}$ & owl:sameAs & 196 & $\mathrm{~L}$ \\
\hline Project Gutenberg & owl:sameAs & 2500 & $S$ \\
\hline Italian Public Schools & owl:sameAs & 5800 & $\mathrm{~S}$ \\
\hline LinkedGeoData & owl:sameAs & 103600 & $\mathrm{~S}$ \\
\hline LinkedMDB & owl:sameAs & 13800 & $\mathrm{~S}$ \\
\hline MusicBrainz & owl:sameAs & 23000 & \\
\hline New York Times & owl:sameAs & 9700 & \\
\hline OpenCyc & owl:sameAs & 27100 & $\mathrm{C}$ \\
\hline OpenEI (Open Energy) & owl:sameAs & 678 & $\mathrm{~S}$ \\
\hline Revyu & owl:sameAs & 6 & \\
\hline Sider & owl:sameAs & 2000 & $\mathrm{~S}$ \\
\hline TCMGeneDIT & owl:sameAs & 904 & \\
\hline UMBEL & rdf:type & 896400 & \\
\hline US Census & owl:sameAs & 12600 & \\
\hline WikiCompany & owl:sameAs & 8300 & \\
\hline WordNet & dbp:wordnet_type & 467100 & \\
\hline YAGO2 & rdf:type & 18100000 & \\
\hline Sum & & 27211732 & \\
\hline
\end{tabular}

lection of vocabularies for marking up content on web pages. The DBpedia 3.8 ontology contains 45 equivalent class and 31 equivalent property links pointing to http://schema.orgterms.
Table 6

Top 10 data sets in Sindice ordered by the number of links to DBpedia.

\begin{tabular}{l|r|r} 
Data set & Link Predicate Count & Link Count \\
\hline okaboo.com & 4 & $2,407,121$ \\
tfri.gov.tw & 57 & 837,459 \\
naplesplus.us & 2 & 212,460 \\
fu-berlin.de & 7 & 145,322 \\
freebase.com & 108 & 138,619 \\
geonames.org & 3 & 125,734 \\
opencyc.org & 3 & 19,648 \\
geospecies.org & 10 & 16,188 \\
dbrec.net & 3 & 12,856 \\
faviki.com & 5 & 12,768
\end{tabular}

\subsection{Incoming Links}

DBpedia is being linked to from a variety of data sets. The overall number of links pointing to DBpedia from other data sets is $39,007,478$ according to the Data $\mathrm{Hub}{ }^{19}$ However, those counts are entered by users and may not always be valid and up-to-date.

In order to identify actually published and online data sets that link to DBpedia, we used Sindice [39]. The Sindice project crawls RDF resources on the web and indexes those resources. In Sindice, a data set is defined by the second-level domain name of the entity's URI, e.g. all resources available at the domain fu-berlin. de are considered to belong to the same data set. A triple is considered to be a link if the data set of subject and object are different. Furthermore, the Sindice data we used for analysis only considers $a u$ thoritative entities: The data set of a subject of a triple must match the domain it was retrieved from, otherwise it is not considered. Sindice computes a graph summary [9] over all resources they store. With the help of the Sindice team, we examined this graph summary to obtain all links pointing to DBpedia. As shown in Table 7. Sindice knows about 248 data sets linking to DBpedia. 70 of those data sets link to DBpedia via Owl: sameAs, but other link predicates are also very common as evident in this table. In total, Sindice has indexed 4 million links pointing to DBpedia. Table 6 lists the 10 data sets which set most links to DBpedia along with the used link predicate and the number of links.

It should be noted that the data in Sindice is not complete, for instance it does not contain all data sets that

\footnotetext{
${ }^{19}$ See http://wiki.dbpedia.org/Interlinking for details.
} 
Table 7

Sindice summary statistics for incoming links to DBpedia.

\begin{tabular}{lr} 
Metric & Value \\
\hline Total links: & $3,960,212$ \\
Total distinct data sets: & 248 \\
Total distinct predicates: & 345
\end{tabular}

Table 8

Top 10 datasets by incoming links in Sindice.

\begin{tabular}{l|r|r} 
domain & datasets & links \\
\hline purl.org & 498 & $6,717,520$ \\
dbpedia.org & 248 & $3,960,212$ \\
creativecommons.org & 2,483 & $3,030,910$ \\
identi.ca & 1,021 & $2,359,276$ \\
13s.de & 34 & $1,261,487$ \\
rkbexplorer.com & 24 & $1,212,416$ \\
nytimes.com & 27 & $1,174,941$ \\
w3.org & 405 & 658,535 \\
geospecies.org & 13 & 523,709 \\
livejournal.com & 14,881 & 366,025
\end{tabular}

are catalogued by the DataHul 20 However, it crawls for RDFa snippets, converts microformats etc., which are not captured by the DataHub. Despite the inaccuracy, the relative comparison of different datasets can still give us insights. Therefore, we analysed the link structure of all Sindice datasets using the Sindice cluster. Table 8 shows the datasets with most incoming links. Those are authorities in the network structure of the web of data and DBpedia is currently ranked second in terms of incoming links.

\section{DBpedia Usage Statistics}

DBpedia is served on the web in three forms: First, it is provided in the form of downloadable data sets where each data set contains the results of one of the extractors listed in Table 1. Second, DBpedia is served via a public SPARQL endpoint and, third, it provides dereferencable URIs according to the Linked Data principles. In this section, we explore some of the statistics gathered during the hosting of DBpedia over the last two of years.

\subsection{Download Statistics for the DBpedia Data Sets}

DBpedia covers more than 100 languages, but those languages vary with respect to the download popular-

$2 6 \longdiv { \text { http: / /datahub.io/ } }$

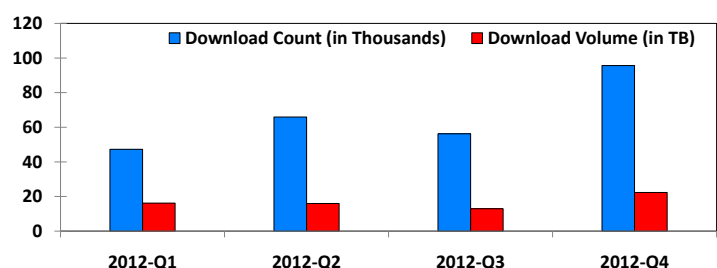

Fig. 9. The download count and download volume (in GB) of the English language of DBpedia.

ity as well. The top five languages with respect to the download volume are English, Chinese, German, Catalan, and French respectively. The download count and download volume of the English language is indicated in Figure 9. To host the DBpedia dataset downloads, a bandwidth of approximately 6 TB per month is currently needed.

Furthermore, DBpedia consists of several data sets which vary with respect to their download popularity. The download count and the download volume of each data set during the year 2012 is depicted in Figure 10 In those statistics we filtered out all IP addresses, which requested a file more than 1000 times per month ${ }^{21}$ Pagelinks are the most downloaded dataset, although they are not semantically rich as they do not reveal which type of links exists between two resources. Supposedly, they are used for network analysis or providing relevant links in user interfaces and downloaded more often as they are not provided via the official SPARQL endpoint.

\subsection{Public Static DBpedia SPARQL Endpoint}

The main public DBpedia SPARQL endpoin 22 is hosted using the Virtuoso Universal Server (Enterprise Edition) version 6.4 software in a 4-nodes cluster configuration. This cluster setup provides parallelization of query execution, even when the cluster nodes are on the same machine, as splitting a query over several nodes allows better use of parallel threads on modern multi-core CPUs on standard commodity hardware.

Virtuoso supports horizontal scale-out, either by redistributing the existing cluster nodes onto multiple machines, or by adding several separate clusters with a round robin HTTP front-end. This allows the cluster setup to grow in line with desired response times for an RDF data set collection. As the size of the DBpedia

\footnotetext{
${ }^{21}$ The IP address was only filtered for that specific file and month in those cases.

22 http://dbpedia.org/sparql
} 


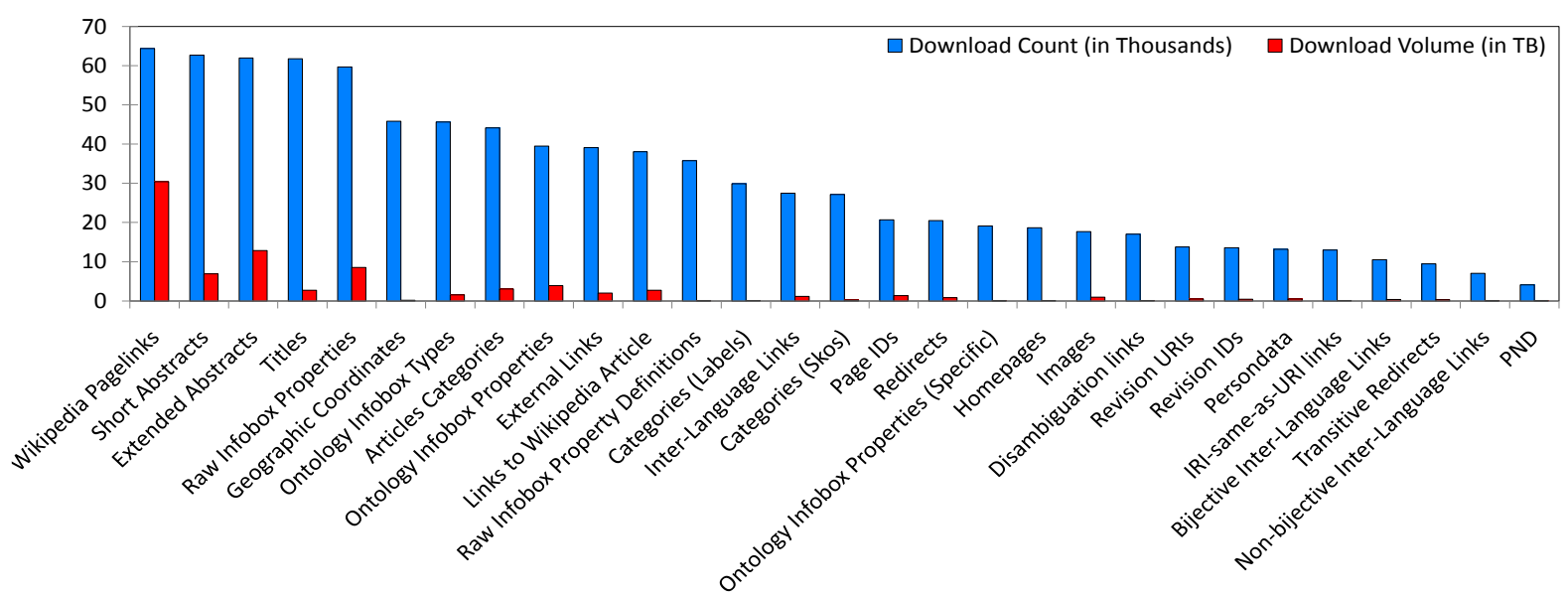

Fig. 10. The download count and download volume (in GB) of the DBpedia data sets.

Table 9

Hardware of the machines serving the public SPARQL endpoint.

\begin{tabular}{ll} 
DBpedia & Configuration \\
\hline $3.3-3.4$ & AMD Opteron 8220 2.80Ghz, 4 Cores, 32GB \\
$3.5-3.7$ & Intel Xeon E5520 2.27Ghz, 8 Cores, 48GB \\
3.8 & Intel Xeon E5-2630 2.30GHz, 8 Cores, 64GB
\end{tabular}

data set increased and its use by the Linked Data community grew, the project migrated to increasingly powerful, but still moderately priced, hardware as shown in Table 9. The Virtuoso instance is configured to process queries within a 1,200 second timeout window and a maximum result set size of 50,000 rows. It provides OFFSET and LIMIT support for paging alongside the ability to produce partial results.

The log files used in the following analysis excluded traffic generated by:

1. clients that have been temporarily rate limited after a burst period,

2. clients that have been banned after misuse,

3. applications, spiders and other crawlers that are blocked after frequently hitting the rate limit or generally use too many resources.

Virtuoso supports HTTP Access Control Lists (ACLs) which allow the administrator to rate limit certain IP addresses or whole IP ranges. A maximum number of requests per second (currently 15) as well as a bandwidth limit per request (currently 10MB) are enforced. If the client software can handle compression, replies are compressed to further save bandwidth. Exception rules can be configured for multiple clients hidden behind a NAT firewall (appearing as a single IP address) or for temporary requests for higher rate
Table 11

Number of unique sites accessing DBpedia endpoints.

\begin{tabular}{lrrrr} 
DBpedia & Avg/Day & Median & Stdev & Maximum \\
\hline 3.3 & 5,824 & 5,977 & 1,046 & 7,865 \\
3.4 & 6,656 & 6,704 & 947 & 8,312 \\
3.5 & 9,392 & 9,432 & 1,643 & 12,584 \\
3.6 & 10,810 & 10,810 & 1,612 & 14,798 \\
3.7 & 17,077 & 17,091 & 6,782 & 33,288 \\
3.8 & 14,513 & 14,493 & 2,783 & 20,510
\end{tabular}

limits. When a client hits an ACL limit, the system reports an appropriate HTTP status code ${ }^{23}$ like 509 and quickly drops the connection. The system further uses an iptables based firewall for permanent blocking of clients identified by their IP addresses.

\subsection{Public Static Endpoint Statistics}

The statistics presented in this section were extracted from reports generated by Webalizer v2.21 24 Table 10 and Table 11 show various DBpedia SPARQL endpoint usage statistics for the DBpedia 3.3 to 3.8 releases. Note that the usage of all endpoints mentioned in Table 12 is counted. The Avg/Day column represents the average number of hits (resp. visits/sites) per day, followed by the Median and Standard Deviation. The last column shows the maximum number of hits (resp. visits/sites) that was recorded on a single day for each data set version. Visits (i.e. sessions of subsequent queries from the same client) are determined by a floating 30 minute

$2 \sqrt[3]{\text { http://en.wikipedia.org/wiki/List_of_HTTP_ }}$ status_codes

${ }^{24}$ http://www.webalizer.org 
Table 10

Number of endpoint hits (left) and visits (right).

\begin{tabular}{lrrrr} 
DBpedia & Avg/Day & Median & Stdev & Maximum \\
\hline 3.3 & 733,811 & 711,306 & 188,991 & $1,319,539$ \\
3.4 & $1,212,549$ & $1,165,893$ & 351,226 & $2,371,657$ \\
3.5 & $1,122,612$ & $1,035,444$ & 386,454 & $2,908,720$ \\
3.6 & $1,328,355$ & $1,286,750$ & 256,945 & $2,495,031$ \\
3.7 & $2,085,399$ & $1,930,728$ & $1,057,398$ & $8,873,473$ \\
3.8 & $2,910,410$ & $2,717,775$ & $1,085,640$ & $7,678,490$
\end{tabular}

\begin{tabular}{lrrrr} 
DBpedia & Avg/Day & Median & Stdev & Maximum \\
\hline 3.3 & 9,750 & 9,775 & 2,036 & 13,126 \\
3.4 & 11,329 & 11,473 & 1,546 & 14,198 \\
3.5 & 16,592 & 16,902 & 2,969 & 23,129 \\
3.6 & 19,471 & 17,664 & 5,691 & 56,064 \\
3.7 & 23,972 & 22,262 & 10,741 & 127,869 \\
3.8 & 16,851 & 16,711 & 2,960 & 27,416
\end{tabular}

Table 12

Hits per service to http://dbpedia.org in thousands.

\begin{tabular}{lrrrrrr} 
Endpoint & 3.3 & 3.4 & 3.5 & 3.6 & 3.7 & 3.8 \\
\hline /data & 1,355 & 2,681 & 2,230 & 2,547 & 3,714 & 4,246 \\
/ontology & 80 & 168 & 142 & 178 & 116 & 97 \\
/page & 2,634 & 4,703 & 1,810 & 1,687 & 3,787 & 7,377 \\
/property & 231 & 311 & 137 & 176 & 176 & 128 \\
/resource & 2,976 & 4,080 & 2,554 & 2,309 & 4,436 & 7,143 \\
/sparql & 2,090 & 4,177 & 2,266 & 9,112 & 15,902 & 15,475 \\
other & 252 & 420 & 342 & 277 & 579 & 695 \\
total & 9,619 & 16,541 & 9,434 & 16,286 & 28,710 & 35,142
\end{tabular}

time window. All requests from behind a NAT firewall are logged under the same external IP address and are therefore counted towards the same visit if they occur within the 30 minute interval.

Table 10 shows the increasing popularity of DBpedia. There is a distinct dip in hits to the SPARQL endpoint in DBpedia 3.5, which is partially due to more strict initial limits for bot-related traffic which were later relaxed. The sudden drop of visits between the 3.7 and the 3.8 data sets can be attributed to:

1. applications starting to use their own private DBpedia endpoint

2. blocking of apps that were abusing the DBpedia endpoint

3. uptake of the language specific DBpedia endpoints and DBpedia Live

\subsection{Query Types and Trends}

The DBpedia server is not only a SPARQL endpoint, but also serves as a Linked Data Hub returning resources in a number of different formats. For each data set we randomly selected 14 days worth of log files and processed those in order to show the various services called. Table 12 shows the number of hits to the various endpoints.

The /resource endpoint uses the Accept: line in the HTTP header sent by the client to return a HTTP sta-

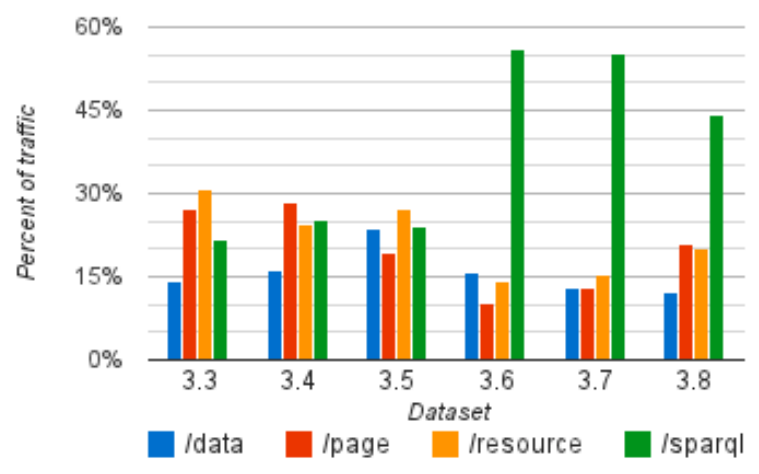

Fig. 11. Traffic Linked Data versus SPARQL endpoint

tus code 30x to redirect the client to either the /page (HTML based) or /data (formats like RDF/XML or Turtle) equivalent of the article. Clients also frequently mint their own URLs to either /page or /data version of an articles directly, or download the raw data directly. This explains why the count of /page and /data hits in the table is larger than the number of hits on the /resource endpoint. The /ontology and /property endpoints return meta information about the DBpedia ontology. While all of these endpoints themselves may use SPARQL queries to generate various page content, these requests are handled by the internal Virtuoso engine directly and do not show up as extra calls to the /sparql endpoint in our analysis.

Figure 11 shows the percentages of traffic hits that were generated by the main endpoints. As we can see, the usage of the SPARQL endpoint has doubled from about 22 percent in 2009 to about 44 percent in 2013. However, this still means that 56 percent of traffic hits are directed to the Linked Data service.

In Table 13, we focussed on the calls to the /sparql endpoint and counted the number of statements per type. As the log files only record the full SPARQL query on a GET request, all the POST requests are counted as unknown.

Finally, we analyzed each SPARQL query and counted the use of keywords and constructs like: 
Table 13

Hits per statement type in thousands.

\begin{tabular}{lrrrrrr} 
Statement & 3.3 & 3.4 & 3.5 & 3.6 & 3.7 & 3.8 \\
\hline ask & 56 & 269 & 360 & 326 & 159 & 677 \\
construct & 55 & 31 & 14 & 11 & 22 & 38 \\
describe & 11 & 8 & 4 & 7 & 62 & 111 \\
select & 1891 & 3663 & 1658 & 8030 & 11204 & 13516 \\
unknown & 78 & 206 & 229 & 738 & 4455 & 1134 \\
total & 2090 & 4177 & 2266 & 9112 & 15902 & 15475
\end{tabular}

Table 14

Trends in SPARQL select (rounded values in \%).

\begin{tabular}{lrrrrrr} 
Statement & 3.3 & 3.4 & 3.5 & 3.6 & 3.7 & 3.8 \\
\hline distinct & 19.5 & 11.4 & 17.3 & 19.4 & 13.3 & 25.4 \\
filter & 45.7 & 13.7 & 31.8 & 25.3 & 29.2 & 36.1 \\
functions & 8.8 & 6.9 & 23.5 & 21.3 & 25.5 & 25.9 \\
geo & 27.7 & 7.0 & 39.6 & 6.7 & 9.3 & 9.2 \\
group & 0.0 & 0.0 & 0.0 & 0.0 & 0.0 & 0.2 \\
limit & 4.6 & 6.5 & 11.6 & 10.5 & 7.7 & 7.8 \\
optional & 30.6 & 23.8 & 47.3 & 26.3 & 16.7 & 17.2 \\
order & 2.3 & 1.3 & 1.9 & 3.2 & 1.2 & 1.6 \\
union & 3.3 & 3.2 & 26.4 & 11.3 & 12.1 & 20.6
\end{tabular}

\section{- DISTINCT}

- FILTER

- FUNCTIONS like CONCAT, CONTAINS, ISIRI

- Use of GEO objects

- GROUP BY

- LIMIT / OFFSET

- OPTIONAL

- ORDER BY

- UNION

For the GEO objects we counted the use of SPARQL PREFIX geo: and $w g s 84^{*}$ : declarations and usage in property tags. Table 14 shows the use of various keywords as a percentage of the total select queries made to the /sparql endpoint for the sample sets. In general, we observed that queries became more complex over time indicating an increasing maturity and higher expectations of the user base.

\subsection{Statistics for DBpedia Live}

Since its official release at the end of June 2011, DBpedia Live attracted a steadily increasing number of users. Furthermore, more users tend to use the synchronisation tool to synchronise their own DBpedia Live mirrors. This leads to an increasing number of live update requests, i.e. changeset downloads. Figure 12 indicates the number of daily SPARQL and synchroni- sation requests sent to DBpedia Live endpoint in the period between August 2012 and January 2013.

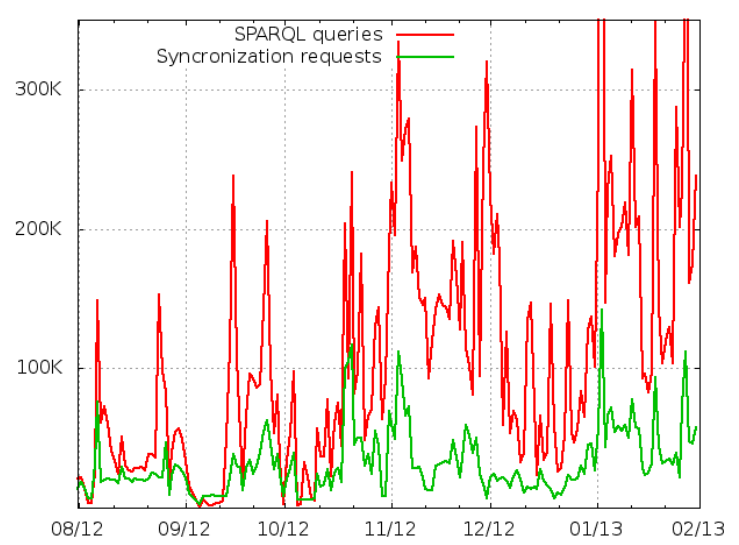

Fig. 12. Number of daily requests sent to the DBpedia Live for a) SPARQL queries and b) synchronisation requests from August 2012 until January 2013

\section{Use Cases and Applications}

Due to DBpedia's coverage of various domains as well as its steady growth as a hub on the Web of Data, the data sets provided by DBpedia can serve many purposes. Such applications include improving search and exploration of Wikipedia, data proliferation for applications, mashups as well as text analysis and annotation tools.

\subsection{Natural Language Processing}

DBpedia can support many tasks in Natural Language Processing (NLP) [33]. For that purpose, DBpedia includes a number of specialized data set 25 For instance, the lexicalizations data set can be used to estimate the ambiguity of phrases, to help select unambiguous identifiers for ambiguous phrases, or to provide alternative names for entities, just to mention a few examples. Topic signatures can be useful in tasks such as query expansion or document summarization, and has been successfully employed to classify ambiguously described images as good depictions of DBpedia entities [13]. The thematic concepts data set of resources can be used for creating a corpus from Wikipedia to be used as training data for topic classifiers, among other

$2 \sqrt[5]{\text { http://wiki.dbpedia.org/Datasets/NLP }}$ 
things (see below). The grammatical gender data set can, for example, be used to add a gender feature in co-reference resolution.

\subsubsection{Annotation: Entity Disambiguation}

An important use case for NLP is annotating texts or other content with semantic information. Named entity recognition and disambiguation - also known as key phrase extraction and entity linking tasks - refers to the task of finding real world entities in text and linking them to unique identifiers. One of the main challenges in this regard is ambiguity: an entity name, or surface form, may be used in different contexts to refer to different concepts. Many different methods have been developed to resolve this ambiguity with fairly high accuracy [22].

As DBpedia reflects a vast amount of structured real world knowledge obtained from Wikipedia, DBpedia URIs can be used as identifiers for the majority of domains in text annotation. Consequently, interlinking text documents with Linked Data enables the Web of Data to be used as background knowledge within documentoriented applications such as semantic search or faceted browsing (cf. Section 7.3).

Many applications performing this task of annotating text with entities in fact use DBpedia entities as targets For example, DBpedia Spotlight [34] is an open source too ${ }^{26}$ including a free web service that detects mentions of DBpedia resources in text. It uses the lexicalizations in conjunction with the topic signatures data set as context model in order to be able to disambiguate found mentions. The main advantage of this system is its comprehensiveness and flexibility, allowing one to configure it based on quality measures such as prominence, contextual ambiguity, topical pertinence and disambiguation confidence, as well as the DBpedia ontology. The resources that should be annotated can be specified by a list of resource types or by more complex relationships within the knowledge base described as SPARQL queries.

There are numerous other NLP APIs that link entities in text to DBpedia: AlchemyAP ${ }^{27}$. Semantic API from Onto $\$^{28}$ Open Calais ${ }^{29}$ and Zemantd ${ }^{30}$ among others. Furthermore, the DBpedia ontology has been used for training named entity recognition systems (without

\footnotetext{
${ }^{26}$ http://spotlight.dbpedia.org/

27 http://www.alchemyapi.com/

28 http://www.ontos.com/

${ }^{29}$ http://www.opencalais.com/

30 http://www.zemanta.com/
}

disambiguation) in the context of the Apache Stanbol projec 31

A related project is ImageSnippet ${ }^{32}$, which is a system for annotating images. It uses DBpedia as one of its main datasets for unambiguously identifying entities depicted within an image.

Tag disambiguation Similar to linking entities in text to DBpedia, user-generated tags attached to multimedia content such as music, photos or videos can also be connected to the Linked Data hub. This has previously been implemented by letting the user resolve ambiguities. For example, Favik ${ }^{33}$ suggests a set of DBpedia entities coming from Zemanta's API and lets the user choose the desired one. Alternatively, similar disambiguation techniques as mentioned above can be utilized to choose entities from tags automatically [14]. The $\mathrm{BBC}^{34}[23]$ employs DBpedia URIs for tagging their programmes. Short clips and full episodes are tagged using two different tools while utilizing DBpedia to benefit from global identifiers that can be easily integrated with other knowledge bases.

\subsubsection{Question Answering}

DBpedia provides a wealth of human knowledge across different domains and languages, which makes it an excellent target for question answering and keyword search approaches. One of the most prominent efforts in this area is the DeepQA project, which resulted in the IBM Watson system [12]. The Watson system won a $\$ 1$ million prize in Jeopardy and relies on several data sets including DBpedia ${ }^{35}$ DBpedia is also the primary target for several QA systems in the Question Answering over Linked Data (QALD) workshop series ${ }^{36}$ Several QA systems, such as TBSL [44], PowerAqua [31], FREyA [1] and QAKiS [7] have been applied to DBpedia using the QALD benchmark questions. DBpedia is interesting as a test case for such systems. Due to its large schema and data size as well as its topic diversity, it provides significant scientific challenges. In particular, it would be difficult to provide capable QA systems for DBpedia based only on simple patterns or via domain specific dictionaries, because of its size and broad coverage. Therefore, a question an-

\footnotetext{
31 http: //stanbol.apache.org/

32 http://wWw. imagesnippets.com

33 http://www.faviki.com/

34 http://bbc.co.uk

35 http://www.aaai.org/Magazine/Watson/

watson.php

http://greententacle.techfak.unibielefeld.de/ cunger/qald/
} 
swering system, which is able to reliable answer questions over DBpedia correctly, could be seen as a truly intelligent system. In the latest QALD series, question answering benchmarks also exploit national DBpedia chapters for multilingual question answering.

Similarly, the slot filling task in natural language processing poses the challenge of finding values for a given entity and property from mining text. This can be viewed as question answering with static questions but changing targets. DBpedia can be exploited for fact validation or training data in this task, as was done by the Watson team [5] and others [28].

\subsection{Digital Libraries and Archives}

In the case of libraries and archives, DBpedia could offer a broad range of information on a broad range of domains. In particular, DBpedia could provide:

- Context information for bibliographic and archive records: Background information such as an author's demographics, a film's homepage or an image could be used to enhance user interaction.

- Stable and curated identifiers for linking: DBpedia is a hub of Linked Open Data. Thus, (re-)using commonly used identifiers could ease integration with other libraries or knowledge bases.

- A basis for a thesaurus for subject indexing: The broad range of Wikipedia topics in addition to the stable URIs could form the basis for a global classification system.

Libraries have already invested both in Linked Data and Wikipedia (and transitively to DBpedia) though the realization of the Virtual International Authority Files (VIAF) project ${ }^{37}$ Recently, it was announced that VIAF added a total of 250,000 reciprocal authority links to Wikipedia ${ }^{38}$ These links are already harvested by DBpedia Live and will also be included in the next static DBpedia release. This creates a huge opportunity for libraries that use VIAF to get connected to DBpedia and the LOD cloud in general.

\subsection{Knowledge Exploration}

Since DBpedia spans many domains and has a diverse schema, many knowledge exploration tools either used DBpedia as a testbed or were specifically built

\footnotetext{
37 http://viaf.org

${ }^{38}$ Accessed on 12/02/2013: http://www.oclc.org/ research/news/2012/12-07a.html
}

for DBpedia. We give a brief overview of tools and structure them in categories:

Facet Based Browsers An award-winning 39 facetbased browser used the Neofonie search engine to combine facts in DBpedia with full-text from Wikipedia in order to compute hierarchical facets [15]. Another facet based browser, which allows to create complex graph structures of facets in a visually appealing interface and filter them is gFacet [16]. A generic SPARQL based facet explorer, which also uses a graph based visualisation of facets, is LODLive [8]. The OpenLink built-in facet based browser ${ }^{40}$ is an interface, which enables developers to explore DBpedia, compute aggregations over facets and view the underlying SPARQL queries.

Search and Querying The DBpedia Query Builder ${ }^{41}$ allows developers to easily create simple SPARQL queries, more specifically sets of triple patterns via intelligent autocompletion. The autocompletion functionality ensures that only URIs, which lead to solutions are suggested to the user. The RelFinder [17] tool provides an intuitive interface, which allows to explore the neighborhood and connections between resources specified by the user. For instance, the user can view the shortest paths connecting certain persons in DBpedia. SemLens [18] allows to create statistical analysis queries and correlations in RDF data and DBpedia in particular.

Spatial Applications DBpedia Mobile [4] is a location aware client, which renders a map of nearby locations from DBpedia, provides icons for schema classes and supports more than 30 languages from various DBpedia language editions. It can follow RDF links to other data sets linked from DBpedia and supports powerful SPARQL filters to restrict the viewed data.

\subsection{Applications of the Extraction Framework: Wiktionary Extraction}

Wiktionary is one of the biggest collaboratively created lexical-semantic and linguistic resources, available in 171 languages (of which approximately 147 can be considered active ${ }^{42}$. It contains information about hundreds of spoken and even ancient languages. In the

36 http://blog.dbpedia.org/2009/11/20/germangovernment-proclaims-faceted-wikipediasearch-one-of-the-365-best-ideas-in-germany/ 4 http://dbpedia.org/fct/

41 http://querybuilder.dbpedia.org/

42 http://s23.org/wikistats/wiktionaries_ html.php 
case of the English Wiktionary there are nearly 3 million detailed descriptions of words covering several domain 43 Such descriptions provide, for a lexical word, a hierarchical disambiguation to its language, part of speech, sometimes etymologies, synonyms, hyponyms, hyperonyms, example sentences, and most prominently senses.

Due to its fast changing nature, together with the fragmentation of the project into Wiktionary language editions (WLE) with independent layout rules a, configurable mediator/wrapper approach is taken for its automated transformation into a structured knowledge base. The workflow of this dedicated Wiktionary extractor being part of the Wiktionary2RDF [19] project is as follows: For every WLE to be transformed an XML configuration file is provided as input. This configuration is used by the Wiktionary extractor, invoked by the DBpedia extraction framework, to first generate a schema reflecting the configured page structure (wrapper part). After this, these language specific schemas are converted to a global schema (mediator part) and later serialized to RDF.

To enable non-programmers (the community of adopters and domain experts) to tailor and maintain the WLE wrappers themselves, a simple XML dialect was created to encode the page structure to be parsed and declare triple patterns, that define how the resulting RDF should be built. The described setup is run against Wiktionary dumps. The resulting data set is open in every aspect and hosted as Linked Data ${ }^{44}$ Statistics are shown in Table 15.

\section{Related Work}

\subsection{Cross Domain Community Knowledge Bases}

\subsubsection{Wikidata}

In March 2012, the Wikimedia Germany e.V. started the development of Wikidate 45 Wikidata is a free knowledge base about the world that can be read and edited by humans and machines alike. It provides data in all languages of the Wikimedia projects, and allows for central access to the data in a similar vein as Wikimedia Commons does for multimedia files. Things described in the Wikidata knowledge base are called items

\footnotetext{
${ }^{43}$ See http://en.wiktionary.org/wiki/semantic for a simple example page

44 http://wiktionary.dbpedia.org/

45 http://wikidata.org/
}

and can have labels, descriptions and aliases in all languages. Wikidata does not aim at offering a single truth about things, but providing statements given in a particular context. Rather than stating that Berlin has a population of 3.5 million, Wikidata contains the statement about Berlin's population being 3.5 million as of 2011 according to the German statistical office. Thus, Wikidata can offer a variety of statements from different sources and dates. As there are potentially many different statements for a given item and property, ranks can be added to statements to define their status (preferred, normal or deprecated). The initial development was divided in three phases:

- The first phase (interwiki links) created an entity base for the Wikimedia projects. This provides a better alternative to the previous interlanguage link system.

- The second phase (infoboxes) gathered infoboxrelated data for a subset of the entities, with the explicit goal of augmenting the infoboxes that are currently widely used with data from Wikidata.

- The third phase (lists) will expand the set of properties beyond those related to infoboxes, and will provide ways of exploiting this data within and outside the Wikimedia projects.

At the time of writing of this article, the development of the third phase is ongoing.

Wikidata already contains 11.95 million items and 348 properties that can be used to describe them. Since March 2013 the Wikidata extension is live on all Wikipedia language editions and thus their pages can be linked to items in Wikidata and include data from Wikidata.

Wikidata also offers a Linked Data interface 46 as well as regular RDF dumps of all its data. The planned collaboration with Wikidata is outlined in Section 9

\subsubsection{Freebase}

Freebase ${ }^{47}$ is a graph database, which also extracts structured data from Wikipedia and makes it available in RDF. Both DBpedia and Freebase link to each other and provide identifiers based on those for Wikipedia articles. They both provide dumps of the extracted data, as well as APIs or endpoints to access the data and allow their communities to influence the schema of the data. There are, however, also major differences be-

\footnotetext{
${ }^{46}$ http://meta.wikimedia.org/wiki/Wikidata/ Development/LinkedDataInterface 4/http://www.freebase.com/
} 
Table 15

Statistical comparison of extractions for different languages.

\begin{tabular}{l|r|r|r|r|r} 
language & \#words & \#triples & \#resources & \#predicates & \#senses \\
\hline en & $2,142,237$ & $28,593,364$ & $11,804,039$ & 28 & 424,386 \\
$\mathrm{fr}$ & $4,657,817$ & $35,032,121$ & $20,462,349$ & 22 & 592,351 \\
$\mathrm{ru}$ & $1,080,156$ & $12,813,437$ & $5,994,560$ & 17 & 149,859 \\
$\mathrm{de}$ & 701,739 & $5,618,508$ & $2,966,867$ & 16 & 122,362
\end{tabular}

tween both projects. DBpedia focuses on being an RDF representation of Wikipedia and serving as a hub on the Web of Data, whereas Freebase uses several sources to provide broad coverage. The store behind Freebase is the GraphD [35] graph database, which allows to efficiently store metadata for each fact. This graph store is append-only. Deleted triples are marked and the system can easily revert to a previous version. This is necessary, since Freebase data can be directly edited by users, whereas information in DBpedia can only indirectly be edited by modifying the content of Wikipedia or the Mappings Wiki. From an organisational point of view, Freebase is mainly run by Google, whereas DBpedia is an open community project. In particular in focus areas of Google and areas in which Freebase includes other data sources, the Freebase database provides a higher coverage than DBpedia.

\subsection{3. $Y A G O$}

One of the projects that pursues similar goals to DBpedia is $Y A G C^{48}$ [42]. YAGO is identical to DBpedia in that each article in Wikipedia becomes an entity in YAGO. Based on this, it uses the leaf categories in the Wikipedia category graph to infer type information about an entity. One of its key features is to link this type information to WordNet. WordNet synsets are represented as classes and the extracted types of entities may become subclasses of such a synset. In the YAGO2 system [21], declarative extraction rules were introduced, which can extract facts from different parts of Wikipedia articles, e.g. infoboxes and categories, as well as other sources. YAGO2 also supports spatial and temporal dimensions for facts at the core of its system.

One of the main differences between DBpedia and YAGO in general is that DBpedia tries to stay very close to Wikipedia and provide an RDF version of its content. YAGO focuses on extracting a smaller number of relations compared to DBpedia to achieve very high precision and consistent knowledge. The two knowledge bases offer different type systems: whereas the DBpedia ontology is manually maintained, YAGO is

\footnotetext{
48 http://www.mpi-inf.mpg.de/yago-naga/yago/
}

backed by WordNet and Wikipedia leaf categories. Due to this, YAGO contains many more classes than DBpedia. Another difference is that the integration of attributes and objects in infoboxes is done via mappings in DBpedia and, therefore, by the DBpedia community itself, whereas this task is facilitated by expert-designed declarative rules in YAGO2.

The two knowledge bases are connected, e.g. DBpedia offers the YAGO type hierarchy as an alternative to the DBpedia ontology and sameAs links are provided in both directions. While the underlying systems are very different, both projects share similar aims and positively complement and influence each other.

\subsection{Knowledge Extraction from Wikipedia}

Since its official start in 2001, Wikipedia has always been the target of automatic extraction of information due to its easy availability, open license and encyclopedic knowledge. A large number of parsers, scraper projects and publications exist. In this section, we restrict ourselves to approaches that are either notable, recent or pertinent to DBpedia. MediaWiki.org maintains an up-to-date list of software projects 49 who are able to process wiki syntax, as well as a list of data extraction extension 50 for MediaWiki.

JWPL (Java Wikipedia Library, [49]) is an opensource, Java-based API that allows to access information provided by the Wikipedia API (redirects, categories, articles and link structure). JWPL contains a MediaWiki Markup parser that can be used to further analyze the contents of a Wikipedia page. Data is also provided as XML dump and is incorporated in the lexical resource UBY ${ }^{51}$ for language tools.

Several different approaches to extract knowledge from Wikipedia are presented in [37]. Given features

\footnotetext{
4 http://www.mediawiki.org/wiki/Alternative_ parsers

st http: //www.mediawiki.org/wiki/Extension Matrix/data_extraction

si http://www.ukp.tu-darmstadt.de/data/ lexical-resources/uby/
} 
like anchor texts, interlanguage links, category links and redirect pages are utilized e.g. for word-sense disambiguations or synonyms, translations, taxonomic relations and abbreviation or hypernym resolution, respectively. Apart from this, link structures are used to build the Wikipedia Thesaurus Web service52. Additional projects that exploit the mentioned features are listed on the Special Interest Group on Wikipedia Mining $(S I G W P)$ Web site 53

An earlier approach to improve the quality of the infobox schemata and contents is described in [47]. The presented methodology encompasses a three step process of preprocessing, classification and extraction. During preprocessing refined target infobox schemata are created applying statistical methods and training sets are extracted based on real Wikipedia data. After assigning a class and the corresponding target schema (classification) the training sets are used to extract target infobox values from the document's text applying machine learning algorithms.

The idea of using structured data from certain markup structures was also applied to other user-driven Web encyclopedias. In [38] the authors describe their effort building an integrated Chinese Linking Open Data (CLOD) source based on the Chinese Wikipedia and the two widely used and large encyclopedias Baidu Baik $£^{54}$ and Hudong Baike 55 Apart from utilizing MediaWiki and HTML Markup for the actual extraction, the Wikipedia interlanguage links were used to link the CLOD source to the English DBpedia.

A more generic approach to achieve a better crosslingual knowledge-linkage beyond the use of Wikipedia interlanguage links is presented in [45]. Focusing on wiki knowledge bases the authors introduce their solution based on structural properties like similar linkage structures, the assignment to similar categories and similar interests of the authors of wiki documents in the considered languages. Since this approach is languagefeature-agnostic it is not restricted to certain languages.

KnowItAl ${ }^{56}$ is a web scale knowledge extraction effort, which is domain-independent, and uses generic extraction rules, co-occurrence statistics and Naive Bayes classification [11]. Cyc [29] is a large com-

${ }^{52}$ http://sigwp.org/en/index.php/Wikipedia_

Thesaurus
53 http://sigwp.org/en/
54 http://baike.baidu.com/
55 http://www.hudong.com/
56 http://www.cs.washington.edu/research/
knowitall/

mon sense knowledge base, which is now partially released as OpenCyc and also available as an OWL ontology. OpenCyc is linked to DBpedia, which provides an ontological embedding in its comprehensive structures. WikiTaxonomy [40] is a large taxonomy derived from categories in Wikipedia by classifying categories as instances or classes and deriving a subsumption hierarchy. The KOG system [46] refines existing Wikipedia infoboxes based on machine learning techniques using both SVMs and a more powerful jointinference approach expressed in Markov Logic Networks. KYLIN [47] is a system which autonomously extracts structured data from Wikipedia and uses selfsupervised linking. Auer et al. [3] introduced an infobox extraction approach for Wikipedia, which later became the DBpedia project.

\section{Conclusions and Future Work}

In this system report, we presented an overview on recent advances of the DBpedia community project. The technical innovations described in this article included in particular: (1) the extraction based on the community-curated DBpedia ontology, (2) the live synchronisation of DBpedia with Wikipedia and DBpedia mirrors through update propagation, and (3) the facilitation of the internationalisation of DBpedia. As a result, we demonstrated that in the past four years DBpedia matured and improved significantly in terms of coverage, usability, and data quality.

With DBpedia, we also aim to provide a proofof-concept and blueprint for the feasibility of largescale knowledge extraction from crowd-sourced content repositories. There are a large number of further crowd-sourced content repositories and DBpedia already had an impact on their structured data publishing and interlinking. Two examples are Wiktionary with the Wiktionary extraction [19] meanwhile becoming part of DBpedia and LinkedGeoData [41], which aims to implement similar data extraction, publishing and linking strategies for OpenStreetMaps.

In the future, we see in particular the following directions for advancing the DBpedia project:

Multilingual data integration and fusion. An area, which is still largely unexplored is the integration and fusion between different DBpedia language editions. Non-English DBpedia editions comprise a better and different coverage of local culture. When we are able to precisely identify equivalent, overlapping and complementary parts in different DBpedia language editions, 
we can reach significantly increased coverage. On the other hand, comparing the values of a specific property between different language editions will help us to spot extraction errors as well as wrong or outdated information in Wikipedia.

Community-driven data quality improvement. In the future, we also aim to engage a larger community of DBpedia users in feedback loops, which help us to identify data quality problems and corresponding deficiencies of the DBpedia extraction framework. By constantly monitoring the data quality and integrating improvements into the mappings to the DBpedia ontology as well as fixes into the extraction framework, we aim to demonstrate that the Wikipedia community is not only capable of creating the largest encyclopedia, but also the most comprehensive and structured knowledge base. With the DBpedia quality evaluation campaign [48] we were making a first step in this direction.

Inline extraction. Currently DBpedia extracts information primarily from templates. In the future, we envision to also extract semantic information from typed links. Typed links is a feature of Semantic MediaWiki, which was backported and implemented as a very lightweight extension for MediaWik ${ }^{57}$. If this extension is deployed at Wikipedia installations, this opens up completely new possibilities for more finegrained and non-invasive knowledge representations and extraction from Wikipedia.

Collaboration between Wikidata and DBpedia. While DBpedia provides a comprehensive and current view on entity descriptions extracted from Wikipedia, Wikidata offers a variety of factual statements from different sources and dates. One of the richest sources of DBpedia are Wikipedia infoboxes, which are structured but at the same time heterogeneous and nonstandardized (thus making the extraction error prone in certain cases). The aim of Wikidata is to populate infoboxes automatically from a centrally managed, highquality fact database. In this regard, both projects complement each other and there are several ongoing collaboration activities. In future versions, DBpedia will include more raw data provided by Wikidata and add services such as Linked Data/SPARQL endpoints, RDF dumps, linking and ontology mapping for Wikidata.

Feedback for Wikipedia. A promising prospect is that DBpedia can help to identify misrepresentations, errors and inconsistencies in Wikipedia. In the future, we plan

57 http://www.mediawiki.org/wiki/Extension: LightweightRDFa to provide more feedback to the Wikipedia community about the quality of Wikipedia. This can, for instance, be achieved in the form of sanity checks, which are implemented as SPARQL queries on the DBpedia Live endpoint, which identify data quality issues and are executed in certain intervals. For example, a query could check that the birthday of a person must always be before the death day or spot outliers that differ significantly from the range of the majority of the other values. In case a Wikipedia editor makes a mistake or typo when adding such information to a page, this could be automatically identified and provided as feedback to Wikipedians.

Integrate DBpedia and NLP. Despite recent advances (cf. Section 7), there is still a huge potential for employing Linked Data background knowledge in various Natural Language Processing (NLP) tasks. One very promising research avenue in this regard is to employ DBpedia as structured background knowledge for named entity recognition and disambiguation. Currently, most approaches use statistical information such as co-occurrence for named entity disambiguation. However, co-occurrence is not always easy to determine (depends on training data) and update (requires recomputation). With DBpedia and in particular DBpedia Live, we have comprehensive and evolving background knowledge comprising information on the relationship between a large number of real-world entities. Consequently, we can employ this information for deciding to what entity a certain surface form should be mapped.

\section{Acknowledgment}

We would like to thank and acknowledge the support of the following people and organisations to DBpedia:

- all Wikipedia contributors

- all DBpedia Mappings Wiki contributors

- OpenLink Software for providing and maintaining the server infrastructure for the main DBpedia endpoint.

- Kingsley Idehen for SPARQL and Linked Data hosting and community support

- Christopher Sahnwaldt for DBpedia development and release management

- Claus Stadler for DBpedia development

- Paul Kreis for DBpedia development

- people who helped contributing data for certain parts of the article:

* Instance Data Analysis: Volha Bryl (working at University of Mannheim) 
* Sindice analysis: Stphane Campinas, Szymon Danielczyk, and Gabriela Vulcu (working at DERI)

* Freebase: Shawn Simister, and Tom Morris (working at Freebase)

This work was supported by grants from the European Union's 7th Framework Programme provided for the projects LOD2 (GA no. 257943), GeoKnow (GA no. 318159) and Dicode (GA no. 257184).

\section{Appendix}

Table 16

List of namespace prefixes.

\begin{tabular}{ll} 
Prefix & Namespace \\
\hline dbo & http://dbpedia.org/ontology/ \\
dbp & http://dbpedia.org/property/ \\
dbr & http://dbpedia.org/resource/ \\
dbr-de & http://de.dbpedia.org/resource/ \\
dc & http://purl.org/dc/elements/1.1/ \\
foaf & http://xmlns.com/foaf/0.1/ \\
geo & http://www.w3.org/2003/01/geo/wgs84_pos\# \\
georss & http://www.georss.org/georss/ \\
ls & http://spotlight.dbpedia.org/scores/ \\
lx & http://spotlight.dbpedia.org/lexicalizations/ \\
owl & http://www.w3.org/2002/07/owl\# \\
rdf & http://www.w3.org/1999/02/22-rdf-syntax-ns\# \\
rdfs & http://www.w3.org/2000/01/rdf-schema\# \\
skos & http://www.w3.org/2004/02/skos/core\# \\
sptl & http://spotlight.dbpedia.org/vocab/ \\
xsd & http://www.w3.org/2001/XMLSchema\#
\end{tabular}

\section{References}

[1] FREyA: An interactive way of querying linked data using natural language.

[2] S. Auer, C. Bizer, G. Kobilarov, J. Lehmann, R. Cyganiak, and Z. Ives. DBpedia: A Nucleus for a Web of Open Data. In Proceedings of the 6th International Semantic Web Conference (ISWC), volume 4825 of Lecture Notes in Computer Science, pages 722-735. Springer, 2008

[3] S. Auer and J. Lehmann. What have Innsbruck and Leipzig in common? extracting semantics from wiki content. In Proceedings of the ESWC (2007), volume 4519 of Lecture Notes in Computer Science, pages 503-517, Berlin / Heidelberg, 2007. Springer.

[4] C. Becker and C. Bizer. Exploring the geospatial Semantic Web with DBpedia Mobile. J. Web Sem, 7(4):278-286, 2009.
Table 17

DBpedia timeline.

\begin{tabular}{|c|c|c|}
\hline Year & Month & Event \\
\hline 2006 & Nov & Start of infobox extraction from Wikipedia \\
\hline \multirow[t]{5}{*}{2007} & Mar & DBpedia 1.0 release \\
\hline & Jun & ESWC DBpedia article [3] \\
\hline & Nov & DBpedia 2.0 release \\
\hline & Nov & ISWC DBpedia article [2] \\
\hline & Dec & DBpedia 3.0 release candidate \\
\hline \multirow[t]{3}{*}{2008} & Feb & DBpedia 3.0 release \\
\hline & Aug & DBpedia 3.1 release \\
\hline & Nov & DBpedia 3.2 release \\
\hline \multirow[t]{2}{*}{2009} & Jan & DBpedia 3.3 release \\
\hline & Sep & JWS DBpedia article 6 \\
\hline \multirow[t]{5}{*}{2010} & Feb & Information extraction framework in Scala \\
\hline & & Mappings Wiki release \\
\hline & Mar & DBpedia 3.4 release \\
\hline & Apr & DBpedia 3.5 release \\
\hline & May & Start of DBpedia Internationalization effort \\
\hline \multirow[t]{4}{*}{2011} & Feb & DBpedia Spotlight release \\
\hline & Mar & DBpedia 3.6 release \\
\hline & Jul & DBpedia Live release \\
\hline & Sep & DBpedia 3.7 release (with $\mathrm{I} 18 \mathrm{n}$ datasets) \\
\hline \multirow[t]{2}{*}{2012} & Aug & DBpedia 3.8 release \\
\hline & Sep & $\begin{array}{l}\text { Publication of DBpedia Internationalization } \\
\text { article } 24\end{array}$ \\
\hline 2013 & Sep & DBpedia 3.9 release \\
\hline
\end{tabular}

[5] D. Bikel, V. Castelli, R. Florian, and D.-J. Han. Entity linking and slot filling through statistical processing and inference rules. In Proceedings TAC Workshop, 2009.

[6] C. Bizer, J. Lehmann, G. Kobilarov, S. Auer, C. Becker, R. Cyganiak, and S. Hellmann. DBpedia - a crystallization point for the Web of Data. Journal of Web Semantics, 7(3):154-165, 2009.

[7] E. Cabrio, J. Cojan, A. P. Aprosio, B. Magnini, A. Lavelli, and F. Gandon. QAKiS: an open domain QA system based on relational patterns. In ISWC-PD; International Semantic Web Conference (Posters \& Demos), volume 914 of CEUR Workshop Proceedings. CEUR-WS.org, 2012.

[8] D. V. Camarda, S. Mazzini, and A. Antonuccio. Lodlive, exploring the Web of Data. In V. Presutti and H. S. Pinto, editors, I-SEMANTICS 2012 - 8th International Conference on Semantic Systems, I-SEMANTICS '12, Graz, Austria, September 5-7, 2012, pages 197-200. ACM, 2012.

[9] S. Campinas, T. E. Perry, D. Ceccarelli, R. Delbru, and G. Tummarello. Introducing RDF graph summary with application to assisted SPARQL formulation. In Database and Expert Systems Applications (DEXA), 2012 23rd International Workshop on, pages 261-266. IEEE, 2012.

[10] O. Erling and I. Mikhailov. RDF support in the Virtuoso DBMS. In S. Auer, C. Bizer, C. Müller, and A. V. Zhdanova, editors, CSSW, volume 113 of LNI, pages 59-68. GI, 2007.

[11] O. Etzioni, M. Cafarella, D. Downey, S. Kok, A.-M. Popescu, T. Shaked, S. Soderland, D. S. Weld, and A. Yates. Web-scale 
information extraction in KnowitAll. In Proceedings of the 13th international conference on World Wide Web, pages 100-110. ACM, 2004.

[12] D. Ferrucci, E. Brown, J. Chu-Carroll, J. Fan, D. Gondek, A. A. Kalyanpur, A. Lally, J. W. Murdock, E. Nyberg, J. Prager, et al. Building Watson: An overview of the DeepQA project. AI magazine, 31(3):59-79, 2010.

[13] A. García-Silva, M. Jakob, P. N. Mendes, and C. Bizer. Multipedia: Enriching DBpedia with multimedia information. In Proceedings of the sixth international conference on Knowledge capture, K-CAP '11, pages 137-144, New York, NY, USA, 2011. ACM

[14] A. García-Silva, M. Szomszor, H. Alani, and O. Corcho. Preliminary results in tag disambiguation using DBpedia. In 1st International Workshop in Collective Knowledage Capturing and Representation (CKCaR), California, USA, 2009.

[15] R. Hahn, C. Bizer, C. Sahnwaldt, C. Herta, S. Robinson, M. Bürgle, H. Düwiger, and U. Scheel. Faceted Wikipedia Search. In W. Abramowicz and R. Tolksdorf, editors, Business Information Systems, 13th International Conference, BIS 2010, Berlin, Germany, May 3-5, 2010. Proceedings, volume 47 of Lecture Notes in Business Information Processing, pages 1-11. Springer, 2010.

[16] P. Heim, T. Ertl, and J. Ziegler. Facet Graphs: Complex semantic querying made easy. In Proceedings of the 7th Extended Semantic Web Conference (ESWC 2010), volume 6088 of LNCS, pages 288-302, Berlin/Heidelberg, 2010. Springer.

[17] P. Heim, S. Hellmann, J. Lehmann, S. Lohmann, and T. Stegemann. RelFinder: Revealing relationships in RDF knowledge bases. In T.-S. Chua, Y. Kompatsiaris, B. Mérialdo, W. Haas, G. Thallinger, and W. Bailer, editors, Semantic Multimedia, 4th International Conference on Semantic and Digital Media Technologies, SAMT 2009, Graz, Austria, December 2-4, 2009, Proceedings, volume 5887 of Lecture Notes in Computer Science, pages 182-187. Springer, 2009

[18] P. Heim, S. Lohmann, D. Tsendragchaa, and T. Ertl. SemLens: Visual analysis of semantic data with scatter plots and semantic lenses. In C. Ghidini, A.-C. N. Ngomo, S. N. Lindstaedt, and T. Pellegrini, editors, Proceedings the 7th International Conference on Semantic Systems, I-SEMANTICS 2011, Graz, Austria, September 7-9, 2011, ACM International Conference Proceeding Series, pages 175-178. ACM, 2011.

[19] S. Hellmann, J. Brekle, and S. Auer. Leveraging the Crowdsourcing of Lexical Resources for Bootstrapping a Linguistic Data Cloud. In JIST, 2012.

[20] S. Hellmann, C. Stadler, J. Lehmann, and S. Auer. DBpedia Live Extraction. In Proc. of 8th International Conference on Ontologies, DataBases, and Applications of Semantics (ODBASE), volume 5871 of Lecture Notes in Computer Science, pages 1209-1223, 2009.

[21] J. Hoffart, F. M. Suchanek, K. Berberich, and G. Weikum. YAGO2: a spatially and temporally enhanced knowledge base from Wikipedia. Artif. Intell, 194:28-61, 2013.

[22] H. Ji, R. Grishman, H. T. Dang, K. Griffitt, and J. Ellis. Overview of the TAC 2010 knowledge base population track. In Third Text Analysis Conference (TAC 2010), 2010.

[23] G. Kobilarov, T. Scott, Y. Raimond, S. Oliver, C. Sizemore, M. Smethurst, C. Bizer, and R. Lee. Media meets Semantic Web - how the BBC uses DBpedia and linked data to make connections. In The semantic web: research and applications, pages 723-737. Springer, 2009.
[24] D. Kontokostas, C. Bratsas, S. Auer, S. Hellmann, I. Antoniou, and G. Metakides. Internationalization of Linked Data: The case of the Greek DBpedia Edition. Web Semantics: Science, Services and Agents on the World Wide Web, 15(0):51 - 61, 2012.

[25] D. Kontokostas, A. Zaveri, S. Auer, and J. Lehmann. TripleCheckMate: A Tool for Crowdsourcing the Quality Assessment of Linked Data. In Proceedings of the 4th Conference on Knowledge Engineering and Semantic Web, 2013.

[26] P. Kreis. Design of a quality assessment framework for the DBpedia knowledge base. Master's thesis, Freie Universität Berlin, 2011.

[27] C. Lagoze, H. V. de Sompel, M. Nelson, and S. Warner The open archives initiative protocol for metadata harvesting. http://www.openarchives.org/OAI/ openarchivesprotocol.html 2008.

[28] J. Lehmann, S. Monahan, L. Nezda, A. Jung, and Y. Shi. LCC approaches to knowledge base population at TAC 2010. In Proceedings TAC Workshop, 2010.

[29] D. B. Lenat. CYC: A large-scale investment in knowledge infrastructure. Communications of the ACM, 38(11):33-38, 1995.

[30] C.-Y. Lin and E. H. Hovy. The automated acquisition of topic signatures for text summarization. In Proceedings of the 18th conference on Computational linguistics, pages 495-501, 2000.

[31] V. Lopez, M. Fernández, E. Motta, and N. Stieler. PowerAqua: Supporting users in querying and exploring the Semantic Web. Semantic Web, 3(3):249-265, 2012.

[32] M. Martin, C. Stadler, P. Frischmuth, and J. Lehmann. Increasing the financial transparency of European Commission project funding. Semantic Web Journal, Special Call for Linked Dataset descriptions, 2013.

[33] P. N. Mendes, M. Jakob, and C. Bizer. DBpedia for NLP - a multilingual cross-domain knowledge base. In Proceedings of the International Conference on Language Resources and Evaluation (LREC), Istanbul, Turkey, 2012.

[34] P. N. Mendes, M. Jakob, A. García-Silva, and C. Bizer. DBpedia Spotlight: Shedding light on the web of documents. In Proceedings of the 7th International Conference on Semantic Systems (I-Semantics), Graz, Austria, 2011.

[35] S. M. Meyer, J. Degener, J. Giannandrea, and B. Michener. Optimizing schema-last tuple-store queries in GraphD. In A. K. Elmagarmid and D. Agrawal, editors, SIGMOD Conference, pages 1047-1056. ACM, 2010.

[36] M. Morsey, J. Lehmann, S. Auer, C. Stadler, and S. Hellmann. DBpedia and the Live Extraction of Structured Data from Wikipedia. Program: electronic library and information systems, 46:27, 2012

[37] K. Nakayama, M. Pei, M. Erdmann, M. Ito, M. Shirakawa, T. Hara, and S. Nishio. Wikipedia mining: Wikipedia as a corpus for knowledge extraction. In Annual Wikipedia Conference (Wikimania), 2008.

[38] X. Niu, X. Sun, H. Wang, S. Rong, G. Qi, and Y. Yu. Zhishi.me: Weaving chinese linking open data. In 10th International Conference on The semantic web - Volume Part II, pages 205-220, Berlin, Heidelberg, 2011. Springer-Verlag.

[39] E. Oren, R. Delbru, M. Catasta, R. Cyganiak, H. Stenzhorn, and G. Tummarello. Sindice.com: A document-oriented lookup index for open linked data. Int. J. of Metadata and Semantics and Ontologies, 3:37-52, Nov. 102008. 
[40] S. P. Ponzetto and M. Strube. WikiTaxonomy: A large scale knowledge resource. In M. Ghallab, C. D. Spyropoulos, N. Fakotakis, and N. M. Avouris, editors, ECAI, volume 178 of Frontiers in Artificial Intelligence and Applications, pages 751-752. IOS Press, 2008.

[41] C. Stadler, J. Lehmann, K. Höffner, and S. Auer. LinkedGeoData: A Core for a Web of Spatial Open Data. Semantic Web Journal, 3(4):333-354, 2012.

[42] F. M. Suchanek, G. Kasneci, and G. Weikum. YAGO: A core of semantic knowledge. In C. L. Williamson, M. E. Zurko, P. F. Patel-Schneider, and P. J. Shenoy, editors, $W W W$, pages 697-706. ACM, 2007.

[43] E. Tacchini, A. Schultz, and C. Bizer. Experiments with Wikipedia cross-language data fusion. In Proceedings of the 5 th Workshop on Scripting and Development for the Semantic Web, ESWC. Citeseer, 2009.

[44] C. Unger, L. Bühmann, J. Lehmann, A.-C. Ngonga Ngomo, D. Gerber, and P. Cimiano. Template-based Question Answering over RDF Data. In Proceedings of the 21st international conference on World Wide Web, pages 639-648, 2012.
[45] Z. Wang, J. Li, Z. Wang, and J. Tang. Cross-lingual Knowledge Linking Across Wiki Knowledge Bases. In Proceedings of the 21st international conference on World Wide Web, pages 459-468, New York, NY, USA, 2012. ACM.

[46] F. Wu and D. Weld. Automatically refining the Wikipedia Infobox Ontology. In Proceedings of the 17th World Wide Web Conference, 2008.

[47] F. Wu and D. S. Weld. Autonomously semantifying Wikipedia In Proceedings of the 16th Conference on Information and Knowledge Management, pages 41-50. ACM, 2007.

[48] A. Zaveri, D. Kontokostas, M. A. Sherif, L. Bühmann, M. Morsey, S. Auer, and J. Lehmann. User-driven Quality Evaluation of DBpedia. In To appear in Proceedings of 9th International Conference on Semantic Systems, I-SEMANTICS '13, Graz, Austria, September 4-6, 2013. ACM, 2013.

[49] T. Zesch, C. Müller, and I. Gurevych. Extracting lexical semantic knowledge from Wikipedia and Wiktionary. In Proceedings of the 6th International Conference on Language Resources and Evaluation, Marrakech, Morocco, May 2008. electronic proceedings. 